\title{
LE SCEPTICISME ET LES HYPOTHÈSES DE LA PHYSIQUE
}

\author{
Sophie Roux
}

Résumé : L'Histoire du scepticisme d'Érasme à Spinoza est souvent invoqué pour appuyer trois thèses : premièrement, Descartes aurait eu une conception dogmatique de la science, et conséquemment de la physique; deuxièmement, l'épistémologie hypothétique de la physique qui se répand au $x v I^{c}$ siècle serait la conséquence d'une crise sceptique générale; troisièmement, cette épistémologie aurait trouvé un terrain plus favorable en Angleterre qu'en France. Ces trois thèses méritent d'être réévaluées : il existe dans l'œuvre cartésienne une tension entre l'idéal d'une science totalement certaine et une physique pleine d'hypothèses; la manière dont les philosophes mécaniques recourent à des hypothèses est indissociable de leur conception de la physique; les physiciens parlent par hypothèses aussi bien en France qu'en Angleterre à la fin du Xvil ${ }^{e}$ siècle. Le problème général abordé ici par le biais du livre de Richard H. Popkin est donc le suivant : dans quelle mesure et pour quelles raisons certaines propositions physiques ont-elles été présentées comme des hypothèses au xvil ${ }^{e}$ siècle?

Mots-CLÉs : Richard H. Popkin, scepticisme, épistémologie de la physique, hypothèse, Descartes, philosophie mécanique.

ABSTRACT : The History of scepticism from Erasmus to Spinoza is often called upon to support three theses : first, that Descartes had a dogmatic notion of systematic knowledge, and therefore of physics; second, that the hypothetical epistemology of physics which spread during the xvith century was the result of a general sceptical crisis; third, that this epistemology was more successful in England than in France. I reject these three theses : I point first to the tension in Descartes' works between the ideal of a completely certain science and a physics replete with hypotheses; further, I argue that the use of hypotheses by mechanical philosophers cannot be separated from their conception of physics; finally I show that, at the end of the xvith century, physicists in France as well as in England spoke through hypotheses and I examine different ways of explaining this shared practice. Richard H. Popkin's book serves therefore as a starting point for insights into the general problem: to what extent and for what reasons some propositions in physics have been presented as hypotheses in the Xvith century?

KErwords : Richard H. Popkin, scepticism, epistemology of physics, hypothesis, Descartes, mechanical philosophy.

Revue de synthèse : $4^{e}$ S. ${ }^{\text {os }}$ 2-3, avr.-sept. 1998, p. 211-255. 
Zusammenfassung: Die Geschichte des Skeptizismus von Erasmus bis Spinoza wird oft benutzt, um drei Thesen zu vertreten: 1 . Descartes häte eine dogmatische Auffassung von Wissenschaft und folglich von Physik gehabt; 2. die Begründung der Physik auf Hypothesen, die sich im 17. Jahrhundert ausbreitete, wäre die Folge einer allgemeinen skeptischen Krise; 3. diese Epistemologie wäre in England auf größere Zustimmung als in Frankreich gestoßen. Nach einer erneuten Auseinandersetzung mit dieser drei Behauptungen läßt sich das Folgende sagen: 1. es existiert im Descartes Werk eine Spannung zwischen dem Ideal einer vollständig sicheren Wissenschaft und einer mit Hypothesen arbeitenden Physik; der Gebrauch von Hypothesen hängt untrennbar mit der Konzeption der Physik der mechanischen Philosophen zusammen; am Ende des 17. Jahrhunderts argumentierten Naturwissenschaftler in Frankreich wie in England mit Hypothesen. Das durch das Buch von Richard H. Popkin angeregte allgemeine Problem, das hier erörtert werden soll, ist damit das folgende : in welchem Maße und aus welchen Gründen sind gewisse physikalische Sätze im 17. Jahrhundert als Hypothesen formuliert worden?

STICHWÖRTER: Richard H.Popkin, Skeptizismus, Begründung der Physik. Hypothese, Descartes, mechanische Philosophie.

Sophie Roux, née en 1965, est agrégée de philosophie et docteur en histoire des sciences. Actuellement chercheur au Max-Planck-Institut für Wissenschaftsgechichte, elle étudie la physique et la philosophie au Xvir siècle.

Adresse : Max-Planck-Institut für Wissenschaftsgechichte, Wilhelmstrasse 44, D-10117 Berlin.

Courrier électronique : roux@mpiwg-berlin.mpg.de 
Les premières recensions ${ }^{\prime}$ de l'Histoire du scepticisme et les recueils ultérieurement consacrés à l'histoire du scepticisme ${ }^{2}$ indiquent rétrospectivement quels furent les deux apports décisifs de cet ouvrage : en montrant la force de la tradition sceptique au XVII siècle, il bouleversait l'appréhension traditionnelle des "grands classiques"; l'existence d'un courant fidéiste détruisait d'autre part l'idée reçue que scepticisme et incroyance religieuse vont toujours main dans la main. Il ne s'agira pas ici de remettre en cause l'un ou l'autre de ces apports, et il serait ridicule, plus de trente ans après que Richard $H$. Popkin a publié son livre, de lui reprocher de n'avoir pas écrit une «Histoire du scepticisme scientifique » qu'il n'a jamais voulu écrire; il est cependant légitime de rappeler que la perspective qui était la sienne excluait par définition d'autres perspectives possibles. L'enquête portera plus spécifiquement sur la catégorie popkinienne de scepticisme modéré : elle a eu un succès si considérable en histoire des sciences qu'elle renvoie indifféremment à la notion de certitude morale, à l'exposition sous forme de dialogue de questions controversées, à la question générale de la certitude des sciences, à l'idée que certains énoncés physiques sont des hypothèses ou à l'apparition du calcul des probabilités. Il s'agira de montrer, à partir d'une question particulière, la diversité épistémique et historique de ces éléments; cette question particulière est celle des hypothèses en physique.

1. Cet article a pour origine une conférence prononcée à l'occasion de la «Journée Popkin » organisée en janvier 1997 par le Centre international de synthèse, le Centre d'études en rhétorique, philosophie et histoire des idées et le Centre Alexandre-Koyré; une version préliminaire a été présentée en septembre 1997 lors d'un séminaire du Max-Planck-Institut für Wissenschaftsgeschichte, Berlin. Je remercie ceux qui ont pris la peine de le lire, et particulièrement Sonja Brentjes, Joan Cadden, Lorraine Daston, Alain Herreman, Didier Piau et Bernhard Thöle. HS renvoie à l'Histoire du scepticisme d'Éasme à Spinoza, trad. de l'anglais par Christine HIVET, présentation de Catherine LaRRère, Paris, Presses universitaires de France (Léviathan), 1995; lorsqu'une œuvre est convoquée pour la première fois, j'en donne les références exhaustives; les sources les plus fréquemment citées sont désignées par leurs initiales en italiques majuscules et les œuvres complètes par une abréviation en majuscules, selon un code explicité au fur et à mesure. Lorsque je ne donne aucune référence à propos de la traduction d'un texte écrit dans une autre langue que le français, je suis responsable de la traduction proposée. "Passim» indique que les références données ne constituent qu'un échantillon des références possibles.

2. Pour les recensions, voir A.-H. Basson, Philosophical Quarterly, 13, 1963, p. 176-178; André-Louis LeROY, Revue philosophique, 154, 1964, p. 115-116; Jacques ROGER, Isis, 52, 1962, p. 514-515; Charles B. SchMIT, Philosophy and phenomenological researches, 23, 1963, p. 455; Julien-Eymard D'ANGERS, Études franciscaines, 1963, p. 238-239. Pour les recueils, The High Road to pyrrhonism, éd. Richard A. Watson et James E. Foce, San Diego, Austin Hill Press, 1980; The Skeptical Tradition, éd. Myles BuRnYEAT, Berkeley/Los Angeles/ Londres, University of California Press, 1983; Scepticism from the Renaissance to the Enlightenment, éd. Richard H. Popkin et Charles B. SchmiTr, Wiesbaden, Otto Harrassowitz, 1987. 
On peut présenter le problème à partir d'une constatation terminologique. Un grand nombre de textes du $x{ }^{\mathrm{e}}{ }^{\mathrm{e}}$ siècle affirment que certains énoncés physiques, qu'il s'agisse de propositions isolées ou des théories qu'elles forment prises toutes ensemble, sont seulement des « hypothèses » ou des « conjectures ", " probables » ou « vraisemblables ». Or ces termes n'ont pas une signification constante d'un texte à l'autre. Dire qu'une proposition est une hypothèse, c'est parfois la stigmatiser comme incertaine (et cela soit parce qu'elle ne porte pas sur des objets immédiatement évidents aux sens ou à la raison, soit parce qu'elle ne peut être déduite d'autres propositions considérées comme évidentes), mais parfois aussi simplement indiquer sa fonction dans une chaîne démonstrative : elle est ce qui est posé en premier et dont on peut déduire d'autres propositions ${ }^{3}$. «Probable » et « vraisemblable » désignent de leur côté une opinion non absolument certaine mais tout de même acceptable, soit parce que des personnes sages et honorables l'ont approuvée, soit parce qu'elle est appuyée par de « bonnes raisons », autrement dit des raisons qui suffisent à emporter la conviction sans être entièrement démonstratives ${ }^{4}$. Peut-on malgré ces différences sémantiques identifier un dénominateur commun minimal des textes en question et construire une catégorie comme celle d'épistémologie hypothétique de la physique? Si ce n'est pas le cas, comment du moins rendre compte de l'introduction du langage des hypothèses en physique? Telle est finalement la question à laquelle cet article doit répondre ${ }^{5}$.

3. La définition du terme «hypothèse » que propose Pierre-Sylvain RéGIs, dans le dictionnaire inséré à la fin de La Physique, in Système de philosophie concernant la logique, la physique et la morale, 3 vol., Amsterdam, 1691, repr. New York/Londres, Johnson Reprint Corporation, 1970, t. I, n. p., est suffisamment générale pour tenir compte de ces différences sémantiques : « HYPOTHĖsE. C'est un mot grec qui signifie supposition. C'est ce qu'on établit pour le fondement de quelque vérité et qui sert à la fois à la faire entendre, soit que la chose qu'on suppose soit vraie, certaine et connue, soit qu'elle soit seulement employée pour expliquer la vérité à laquelle elle se rapporte. "Egalement Antoine FurETıèRE, Essai d'un dictionnaire universel, Paris, 1690, entrée " HYPOTHËSE »: «Supposition qu'on fait d'un principe, d'une proposition, pour en tirer des raisonnements et des conséquences. "

4. Contrairement à ce qu'affirme Ian HACKING, The Emergence of probability, Cambridge, Cambridge University Press, 1975 , chap. Iv, il n'est pas vrai que, jusqu'au xvir siècle, le terme " probable " ait qualifié exclusivement des opinions crédibles parce que approuvées par des autorités. Sur ce point, on se référera en première instance à Daniel GARBER et Sandy $Z_{A B E L}$, On the emergence of probability ", Archive for history of exact sciences, vol. 21, I, 1979, p. 37-47.

5. Pour éviter d'entrée de jeu tout malentendu, j'ajouterai deux choses. En premier lieu, assimiler cette catégorie à ce que certains appellent aujourd'hui méthode hypothético-déductive serait non seulement anachronique mais inutile, car la catégorie de «méthode hypothético-déductive » pose les mêmes problèmes que celle d'« épistémologie hypothétique de la physique ». En second lieu, mon propos n'est pas d'identifier la méthode qui aurait caractérisé la « Science moderne », ou aurait rendu possible son émergence - pour que cette affirmation ait un sens, il faudrait commencer par montrer que quelque chose comme la Science moderne existe, qu'elle est caractérisée par une méthode spécifique, et que c'est cette méthode qui a rendu possible la science en question. 
En un mot, la catégorie de scepticisme modéré que Popkin avait construite dans le contexte d'une histoire du scepticisme n'est pas forcément adaptée à une histoire du statut épistémologique des énoncés physiques au XVII ${ }^{\mathrm{e}}$ siècle; il faut dès lors se demander quelles catégories lui substituer et dans quelle espèce de discours historiques ces catégories opèrent. Nous procédons à cet effet en trois temps. Le schéma interprétatif de Popkin est fondé sur l'opposition entre le dogmatisme de Descartes et le scepticisme de Marin Mersenne et de Pierre Gassendi; nous nous interrogeons en premier lieu sur la portée de cette opposition, car la physique concrète de Descartes recourt comme celle de Gassendi à des hypothèses. En deuxième lieu, alors que Popkin interprète le scepticisme modéré principalement comme une réaction à la crise sceptique générale amorcée au $\mathrm{XvI}^{\mathfrak{e}}$ siècle, nous soutenons que la conception hypothétique de la physique a partie liée avec la manière dont les philosophes mécaniques concevaient l'objet de la physique. Enfin, contre l'idée que le scepticisme modéré a dû se réfugier à la Royal Society faute de trouver un terrain favorable sur le continent, nous examinons dans quelle mesure et pour quelles raisons le discours des hypothèses l'emporte également en France à la fin du XVII siècle.

\section{I. - LES HYPOTHÈSES DE LA PHYSIQUE CARTÉSIENNE}

Rappelons succinctement comment Popkin construit la catégorie de scepticisme modéré. La crise sceptique, initialement occasionnée par des controverses religieuses et avivée par la redécouverte des textes pyrrhoniens, a selon lui provoqué deux réactions chez les savants du début du XvII siècle. Descartes d'un côté, maintenant l'idéal aristotélicien et dogmatique de la science, aurait tenté de retourner le scepticisme contre luimême en tirant d'un doute radical une certitude métaphysique sur laquelle fonder la science. D'un autre côté, Nicolas Claude Fabri de Peiresc, Mersenne, Gilles Personne de Roberval, Pierre de Fermat ou Gassendi, cherchant une voie moyenne entre le scepticisme et le dogmatisme, auraient élaboré une nouvelle conception de la science : elle aurait pour objet les apparences et non la nature des choses; sa modalité serait celle du vraisemblable et du probable, plutôt que de l'évidence et de la certitude. C'est cette conception de la science que Popkin appelle «scepticisme modéré » ou " constructif ${ }^{6}$. En peignant Descartes comme un penseur solitaire, attardé

6. L'expression « scepticisme modéré » trouve son origine dans un passage célèbre de Gassendi : « Pour voir le peu de choses que, parmi toutes ces opinions sur les critères de la vérité, on peut dire avec probabilité, il semble qu'il faille tenir une route intermédiaire entre les sceptiques (par ce terme j'embrasse tous ceux qui nient [l'existence de] critères) et les dog- 
dans la quête vaine d'une certitude absolue, alors qu'une pléiade de savants auraient depuis longtemps compris qu'il fallait se contenter d'hypothèses probables en physique, Popkin ne faisait qu'ajouter une pièce à un dossier aussi vénérable que problématique ${ }^{7}$.

Le Descartes qui intervient dans ce diptyque est en effet celui des Méditations; or, quelle que soit la valeur fondatrice de cet ouvrage, il ne résume pas l'œuvre cartésienne, et, en particulier, ne préjuge pas de la manière dont Descartes pratique la physique ou de la façon dont il la thématise à titre de science particulière. Pour argumenter un instant anhistoriquement, il est rare qu'une science particulière (et plus encore tel énoncé particulier de telle science particulière) soit conforme à la définition idéale de la science; établir les fondations d'une science n'est pas garantir la certitude de chacune de ses propositions; enfin, la théorie de la connaissance d'un philosophe n'indique pas a priori l'espèce de connaissance qu'il espère atteindre dans un domaine particulier du savoir. Autrement dit, les Méditations ne nous disent pas grand-chose sur le statut épistémique des énoncés de la physique cartésienne; or, dans cette physique, un certain nombre d'énoncés sont hypothétiques. Popkin avait disqualifié le problème en affirmant que de tels énoncés étaient seulement « tactiques ${ }^{8} »$. De fait, les précautions cartésiennes qui ménagent manifestement l'enseignement de l'Église sont certainement « tactiques »; mais rien ne permet de généraliser cette interprétation. Pourquoi donc y a-t-il des hypothèses dans la physique cartésienne? Comment concilier leur présence avec l'exigence de certitude propre à la science cartésienne? Dans quelle mesure remettent-elles en question l'opposition entre Descartes et Gassendi?

matiques. » Ce passage se trouve in Pierre GASSENDI, Syntagma philosophicum (cité par la suite comme $S P$ ), pars 1 , lib. 2, cap. 5 : « Posse aliquam veritatem signo aliquo innoscere, et criterio dijudicari ", in Opera Omnia, 6 vol., Lyon, 1658, réimpr. Stuttgart, Friedrich Fromman Verlag, 1964 (cité par la suite comme 0.0.), I 79b. Ce passage est partiellement cité in $H S$, p. 151.

7. C'était déjà sur la dette d' $H S$ envers la littérature secondaire qu'A.-L. Leror concluait le compte rendu cité supra $\mathrm{n}$. 2. De fait, Henri Berr puis Bernard Rochot avaient depuis longtemps entrepris d'évaluer les doses variables de scepticisme et d'épicurisme dans les différents écrits de Gassendi; voir H. BERR, An jure inter scepticos Gassendus numeratus fuerit, Paris, Hachette, 1898, et, parmi les nombreuses publications de B. Rоснот, "Le philosophe », in Pierre Gassendi, 1592-1655. Sa vie et son æeuvre, éd. Centre international de synthèse, Paris, Albin Michel, 1955, p. 71-107. Par la suite, la question avait de nouveau été abordée par Tullio Gregory, Scetticismo ed empirismo, Bari, Laterza, 1961 et par Olivier-René BloCH, La Philosophie de Gassendi, La Haye, Martinus Nijhoff, 1971. De son côté, Robert Lenoble avait opposé le mécanisme métaphysique et dogmatique de Descartes et le mécanisme pragmatiste ou positiviste de Mersenne, Gassendi, Hobbes, Roberval et Pascal; voir R. LENoBle, in Mersenne ou la naissance du mécanisme, Paris, Vrin, 1943, en part., chap. XI : « Le mécanisme positif. "

8. Voir HS, p. $200:$ Galilée, Campanella et Descartes [o]nt parfois affirmé, pour des raisons tactiques, que leurs théories étaient avant tout hypothétiques. » 
Cette première partie n'a pas pour objet de nier l'existence d'un différend entre Descartes et Gassendi, mais d'en préciser la portée : Descartes reconnaît comme Gassendi le caractère hypothétique des explications particulières de la physique. Sans procéder ici à une exégèse systématique des trois exposés que Descartes a donnés de sa physique, les Essais (ici la Dioptrique et les Météores), le Monde et les Principes, nous nous concentrerons sur le dernier de ces ouvrages, que Descartes lui-même donne comme le plus complet des trois ${ }^{9}$. Nous commençons par établir l'existence d'hypothèse dans les Principes et nous en précisons le statut. Nous signalons ensuite que l'emploi d'hypothèses en physique ne permet pas de conclure à l'identité épistémologique des physiques de Descartes et de Gassendi. Nous étudions enfin les procédures mises en place à titre provisoire dans les Essais : elles sont toujours à l'œuvre dans les Principes et l'ultime tentative de Descartes pour surmonter la tension entre l'exigence de certitude qui caractérise selon lui la science et la pratique des hypothèses est la notion de système.

La fréquence des termes hypothétiques dans les livres physiques des Principes est élevée; un premier examen de ce champ sémantique, limité aux expressions verbales, appelle deux remarques ${ }^{10}$. En premier lieu, la version française recourt bien plus souvent que la version latine au mode hypothétique et elle efface les différences que cette dernière faisait entre différentes espèces d'hypothèses. D'une part, en effet, elle introduit le terme " supposer » sans aucun correspondant latin ${ }^{11}$. D'autre part, elle traduit indifféremment par "supposer» "arbitror », " assumo », " existimor ", " excogito ", " hypothesin propono ", "imaginor ", "fingo ", " pono », " puto » ou " suppono ", alors que l'usage de ces verbes était dif-

9. Sur la notion d'hypothèse dans la physique de Descartes, voir en général Monique MARTINET, "Science et hypothèses chez Descartes », Archives internationales d'histoire des sciences, vol. 24, 95, 1974 et Desmond M. CLARKE, Descartes' philosophy of science, Manchester, Manchester University Press, 1982. À titre complémentaire, voir Larry LAUDAN, « The Clock metaphor and probabilism ", Annals of science, 22, 1966, repr. in Science and hypothesis. Historical essays on scientific methodology, Dordrecht, D. Reidel Publishing Company, 1981; A. I. SABRA, Theories of light from Descartes to Newton, Londres, Oldboume, 1967; Eman McMuldin, "Conceptions of science in the Scientific Revolution », in Reappraisals of the Scientific Revolution, éd. David C. LindBerg et Robert S. Westman, Cambridge, Cambridge University Press, 1990; passim.

10. Ce premier examen suffisait à mon propos; l'index établi in Franco Aurelio MEsCHINI, Indice dei « Principia philosophiae » di René Descartes, Florence, Leo S. Olchski, 1996, permettrait son étude systématique.

11. Par ex. Descartes, Principes de la philosophie, III 68, III 73, III 74, III 118. Dans ce qui suit, les Principes de la philosophie sont cités comme PP; l'édition choisie est Descartes. Euvres complètes, éd. Charles ADAM et Paul TANNERY, 11 vol., nouv. présentation, Bernard Rochot et Pierre Costabel, Paris, Vrin, 1964-1974, citée par la suite comme A. T.; je donne dans le corps du texte la version française et signale en note les modifications qu'elle introduit par rapport au texte latin. 
férencié et normé en latin ${ }^{12}$. (Par exemple, «fingo" désigne des fictions peu vraisemblables, voire fausses ${ }^{13}$; «suppono» est le verbe privilégié pour désigner les suppositions générales avancées à l'issue de la description des principaux phénomènes astronomiques ${ }^{14}$; « hypothesis » enfin est le terme réservé pour les trois systèmes du monde ${ }^{15}$ et plus généralement pour des propositions assumées pour leur pouvoir explicatif plutôt que pour leur vérité ${ }^{16}$.) En second lieu, l'absence de termes hypothétiques ne constitue pas un indice absolu de la certitude d'une proposition: les termes signalant explicitement des hypothèses sont moins fréquents dans le livre IV que dans le livre III, non parce que les explications de celui-là sont plus certaines que celles de celui-ci, mais sans doute parce qu'elles sont présentées comme des conséquences, indubitables à titre de conséquences, des suppositions générales de l'article III 46.

La fréquence de ce vocabulaire hypothétique étonnera peut-être. On connaît, en effet, la décision initiale qui fonde l'entreprise cartésienne : assimiler le probable au faux et réserver le beau nom de science à ce qui est évident par intuition ou certain par déduction ${ }^{17}$. Plus précisément, les Principes entendent donner à la science, et en particulier à la physique, une fondation métaphysique : Descartes affirme en ce sens que sa métaphysique contient ou appuie les principes de sa physique, qu'elle permet de les déduire, de les prouver ou de les démontrer ${ }^{18}$. Un tel étonnement sera cependant tempéré par les deux remarques suivantes. D'une part, la déci-

12. Par ex. PP III 20, III 46, III 77, III 80 , III 123, III 126. Également les références données à la note suivante.

13. Par ex. $P P$ III 19, III 36, III 61, III 70, III 87, III 155, IV 1 , IV 2.

14. Par ex. PP III 46, III 47, III 83, III 85, III 88. PP III 46, in A.T. IX-2, p. 125 permet de préciser que les suppositions générales en question consistent à poser l'existence de tourbillons dans le monde qui est le nôtre : Dieu a divisé la matière en parties égales entre elles et de grosseur médiocre; il les a fait se mouvoir de deux façons, "chacune à part autour de son propre centre [... ], et avec cela plusieurs ensemble autour de quelques centres ».

15. En part. de $P P$ III 15 à 19 et de $P P$ III 37 à 40.

16. L'« hypothesis » des premières lignes de $P P$ III 48 reprend la «falsa suppositio » de la fin de $P P$ III 47. $P P$ IV 1-2 renvoie à ce même passage comme à une « falsa hypothesis ». Cet usage est conforme à la définition d'« hypothesis » donnée dans PP III 15, in A.T. VIII-1, p. 85 : « hypotheses, hoc est, positiones, quae non ut verae, sed tantum ut phaenomenis explicandis idoneae, considerantur. »

17. Voir Regulae ad directionem ingenii, regula II, in A.T. X, p. 362 : «Omnia scientia est cognitio certa et evidens [...] Per hanc propositionem rejicimus illas omnes probabiles tantum cognitiones "; regula XIV, in A.T. X, p. 425. Également, Discours de la méthode (cité par la suite comme $D M$ ), première partie, in A.T. VI, p. 8 : « Je réputais presque pour faux tout ce qui n'était que vraisemblable »; quatrième partie, in A.T. VI, p. 31-32. À Mersenne, 5 oct. 1637 , in A.T. I, p. 450 ; passim.

18. On trouve ces différentes formules dans: à Mersenne, 15 avr. 1630, 17 mai 1638, 11 nov. 1640, 28 janv. 1641, 26 avr. 1643 et « Lettre-préface aux Principes », in respectivement A.T. I, p. 258-259, A.T. II, p. 141, A.T. III, p. 233, A.T. III, p. 298, A.T. III, p. 648-649 et A.T. IX-2, p. 19; passim. Leur variété même indique la complexité du rapport entre physique et métaphysique; il est analysé in D. M. CLARKE, op. cit. supra n. 9, p. 77-107; Daniel GARBer, Descartes' metaphysical physics, Chicago, Chicago University Press, 1992, p. 24-26, 
sion fondatrice de Descartes ne l'avait pas empêché de déclarer chimérique une science des choses naturelles totalement déductive - tout en gardant il est vrai une certaine tendresse pour cette chimère ${ }^{19}$. D'autre part, il souligne explicitement dans les Principes que la seule chose que l'on puisse connaître avec une certitude absolue en physique, ce sont certains principes généraux. Bien des commentateurs ont assurément prétendu que le Deus ex machina qui intervient à la fin de cet ouvrage avait pour fonction de garantir la certitude de l'ensemble de la physique. Il est vrai que nous possédons une certitude métaphysique de certaines vérités; mais elle ne s'étend pas à toutes les propositions physiques :

«[...] elle s'étend à toutes les choses qui peuvent être démontrées, touchant ces corps, par les principes de la mathématique ou par d'autres aussi évidents et certains; au nombre desquelles il me semble que celles que j'ai écrites en ce traité doivent être reçues, au moins les principales et les plus générales [...] J'ai prouvé par démonstration mathématique toutes les choses que j'ai écrites, au moins les plus générales qui concernent la fabrique du ciel et de la terre ${ }^{20}$. »

Ces choses générales, que l'on peut savoir d'un savoir vraiment certain car elles sont garanties par la métaphysique, se réduisent à trois thèses : tous les phénomènes s'expliquent par la grandeur, la figure et le mouvement des corps; l'essence de la matière consiste dans l'extension (ce qui implique en particulier l'impossibilité des atomes et du vide); le mouvement obéit à des lois valables dans n'importe quel monde ${ }^{21}$.

52-62; Frédéric de Buzon et Vincent Carraud, Descartes et les "Principia II », Paris, Presses universitaires de France, 1994, p. 28-35, 121-126.

19. Voir à Mersenne, 10 mai 1632, in A.T. I, p. 250-252: « La connaissance de cet ordre [l'ordre régulier et déterminé de toutes les étoiles] est la clef et le fondement de la plus haute et plus parfaite science que les hommes puissent avoir touchant les choses matérielles; d'autant que par son moyen on pourrait connaître a priori toutes les diverses formes et essences des corps terrestres, au lieu que, sans elle, il nous faut nous contenter de les deviner $a$ posteriori, et par leurs effets [...] Je crois que c'est une science qui passe la portée de l'esprit humain; et toutefois je suis si peu sage, que je ne saurais m'empêcher d'y rêver, encore que je juge que cela ne servira qu'à me faire perdre du temps. »

20. PP IV 206, in A.T. IX-2, p. 324-325. Je souligne. Quoique plus concise, la version latine, in A.T. VIII-1, p. 329, s'achève sur la même précision : "[...] his enim admissis, caetera omnia, saltem generaliora quae de mundo et terra scripsi, vix aliter quam a me explicata sunt, intelligi posse videntur. "Egalement à Mersenne, 28 oct. 1640, in A.T. III, p. 212. Dans ces trois textes, Descartes se réserve la possibilité d'étendre la certitude des premiers principes aux explications particulières ( "au moins », " saltem ", les principes généraux sont certains); rien ne prouve cependant qu'il soit, ou qu'il pense être, effectivement en mesure de procéder à cette extension.

21. La démarcation entre les propositions physiques que la métaphysique garantit et celles qu'elle ne garantit pas correspond selon moi à la séparation matérielle entre le deuxième livre des Principes et les deux suivants. Ce qui a au moins deux conséquences : premièrement, la distinction entre les corps durs et les corps mous a le même statut que les lois du mouvement, qui ont outre mesure focalisé l'attention des commentateurs; en second lieu, la séparation de la matière en trois éléments n'est pas une proposition métaphysiquement fondée, pace D. M. ClaRKe, op. cit. supra n. 9, p. 82, passim. Il resterait à thématiser conceptuellement 
Il n'est donc pas question de fonder métaphysiquement l'explication d'un phénomène particulier. Plus précisément, les explications particulières sont des hypothèses seulement probables parce que, à partir des premiers principes, on peut déduire plusieurs explications possibles d'un même phénomène :

«[...]les principes que j'ai ci-dessus expliqués, sont si amples qu'on en peut déduire beaucoup plus de choses que nous n'en voyons dans le monde, et même beaucoup plus que nous n'en saurions parcourir de la pensée en tout le temps de notre vie ${ }^{22}$.»

Ce qui garantit ici la certitude d'une proposition n'est pas seulement la linéarité d'une déduction, mais ce qu'on pourrait appeler son univocité : telle explication d'un phénomène n'est pas certaine parce que les principes généraux que la métaphysique assure ne sont pas suffisamment déterminés pour qu'on puisse la préférer à quantité d'autres explications possibles. Il y a bien un moyen de pallier cette indétermination : l'observation des phénomènes ou la mise en place d'expériences discriminantes permet parfois de choisir entre plusieurs spécifications possibles des principes généraux ${ }^{23}$. Mais lorsque cela n'est pas possible, il faut se contenter de proposer, à titre d'hypothèses, quelques explications possibles :

« Et touchant tels effets particuliers, desquels nous n'avons pas assez d'expériences pour déterminer quelles sont les vraies causes qui les produisent, nous devons nous contenter d'en savoir quelques-unes par lesquelles il se peut faire qu'ils soient produits ${ }^{24}$.»

Nous pouvons donc, à ce point de l'argumentation, présenter les conclusions suivantes : il existe des hypothèses dans les Principes; elles portent sur les explications particulières des phénomènes qui ne peuvent être déduites univoquement des principes généraux de la physique; parfois, mais à en juger par le nombre de propositions présentées comme des hypo-

cette démarcation; il s'agit d'une affaire compliquée, parce que cela revient à rendre compte de la différence entre les causes " générales " et “ particulières » de la physique cartésienne, ce qui suppose une analyse détaillée de la notion de cause.

22. PP III 4, in A.T. IX-2, p. 105. La version française, in A.T. VIII-1, p. 81, est moins emphatique que la version latine : "tam vasta et tam focunda " est rendu par «si amples »; " umquam " par « en tout le temps d'une vie ". Également $D M$, sixième partie, in A.T. VI, p. 64-65.

23. $P P$ III 4 , in A.T. IX-2, p. 105. Également $P P$ III 36, in A.T. IX-2, p. $124 ; D M$, sixième partie, in A.T. VI, p. 64-65.

24. PP III 132, in A.T. IX-2, p. 185. Le latin, in A.T. VIII-1, p. 185, parle explicitement de "causae verisimiles », et c'est la seule fois où Descartes qualifie de «vraisemblable » une explication physique dans les Principes. Également à Huygens, 5 oct. 1646, in A.T. IV, p. 516. 
thèses dans les Principes, cela est finalement rare, il est possible de supprimer cette plurivocité par des expériences. De la présence d'hypothèses dans la physique cartésienne, faut-il conclure à l'identité épistémologique de la physique cartésienne et de la physique gassendiste ${ }^{25}$ ?

Évidemment non. Les principes généraux de la physique n'ont pas de fondement métaphysique selon Gassendi, soit qu'elle n'en ait pas besoin, soit qu'il soit impossible de trouver un tel fondement; et Gassendi ne les présente pas comme des certitudes. L'atomisme par exemple n'est pas tenu pour une vérité, mais seulement pour une hypothèse meilleure que les autres :

«Il peut sembler qu'Épicure me séduise plus que les autres [...] mais je n'approuve pas pour autant toutes ses thèses, même parmi celles qui ne touchent pas à la religion; et celles que j'approuve, je ne les embrasse pas au point que je les tienne pour indubitables et certaines et que je ne juge pas bien plutôt qu'elles demeurent dans le domaine de la vraisemblance ${ }^{26}$. »

Corrélativement, même s'il existe des hypothèses chez Descartes comme chez Gassendi, elles ne sont pas hypothétiques pour les mêmes raisons : la certitude d'une proposition dépend pour le second principalement de son référent sensible, et non de son insertion univoque dans une chaîne déductive. Ainsi affirme-t-il que toutes les sensations sont vraies en tant qu'elles sont immédiates et qu'elles constituent le premier critère de la vérité ${ }^{27}$; la philosophie naturelle est hypothétique, vraisemblable ou conjecturale précisément parce qu'elle consiste à dépasser les sensations immédiates, « juge[ant] des choses qui nous sont cachées d'après celles qui nous sont

25. Outre les publications indiquées supra $\mathrm{n}$. 7, sur Gassendi en général voir Lynn Sumida Joy, Gassendi the atomist. Advocate of history in an age of science, Cambridge, Cambridge University Press, 1987. Barry BRUNDELL, Pierre Gassendi. From aristotelianism to a new natural philosophy, Dordrecht, D. Reidel Publishing Company, 1987. Sur les différentes attitudes épistémologiques de Gassendi, voir en premier lieu O.-R. BLoch, op. cit. supra $\mathrm{n}$. 7, p. 77-109.

26. SP, «Liber proemialis de philosophia universe », in O.O. I 30a-b. Dans le contexte de la polémique contre Descartes, GASSENDI, in Disquisitio metaphysica seu dubitationes et instantiae adversus Renatus Cartesii metaphysicam et responsa (cité par la suite comme $R M$ ), contre la cinquième méditation, doute 1 , art. 1 , in 0.0 . III 376b-377a, et in Recherches métaphysiques, rééd. et trad. par B. Rоснот, Paris, Vrin, 1962 (cité par la suite comme Rochot, 1962), p. 478, souligne l'évidence des principes mécaniques : « [...] que les choses matérielles soient toutes douées de quantité et de parties, de figures et d'une certaine disposition des parties, et d'autres choses de ce genre, c'est ce que même les aveugles et les perruquiers n'ignorent pas. »

27. Voir $R M$, contre la deuxième méditation, doute 1, art. 2, in 0.0 . III 286a et in Rochot, 1962, p. 68; Exercitationes paradoxicae adversus aristoteleos (par la suite DP), livre second, cinquième dissertation, art. 1 et sixième dissertation, art. 1 , in 0.0 . III respectivement $182 \mathrm{~b}$ et $192 \mathrm{~b}$, et in Dissertations en forme de paradoxes contre les aristotéliciens, rééd. et trad. par B. RochOt, Paris, Vrin, 1959 (cité par la suite comme RochOT, 1959), respectivement p. 388 et 436; passim. 
percevables par leurs apparences ${ }^{28} »$. Lorsqu'il défend contre les sceptiques l'existence de signes indiquant démonstrativement ce que sont certaines choses naturellement cachées, Gassendi va jusqu'à parler d'un savoir certain et vrai; mais la source de ce savoir est toujours dans les choses sensibles : la sueur constitue un signe sensible de l'existence de pores dans la peau, le passage de la lumière à travers le verre, un signe sensible de son caractère corpusculaire, le mouvement des corps, un signe sensible de l'existence du vide ${ }^{29}$.

Ce qui suggère deux conclusions. D'une part, théorie de la connaissance et épistémologie de la physique ne vont pas toujours de compagnie - ce qui n'interdit pas leurs rencontres. D'autre part, l'emploi d'hypothèses ne suffit pas à caractériser une épistémologie, même une fois précisé qu'on entend par là l'idée que certains énoncés physiques ne peuvent prétendre à la même certitude que les théorèmes mathématiques, sans pourtant être de pures opinions, que l'on évaluerait d'après le nombre et l'autorité des personnes qui les ont soutenues. C'est dire que la catégorie «épistémologie hypothétique de la physique » est philosophiquement pauvre; dans le troisième et dernier temps de cette première partie, nous donnons conséquemment quelques indications supplémentaires sur l'épistémologie hypothétique de la physique propre à Descartes. Nous examinerons tout d'abord certaines procédures mises en place dans les Essais, c'est-à-dire à un moment où Descartes ne voulait pas exposer la totalité de son système. Un tel détour peut être justifié à titre préliminaire et provisoire par la complémentarité des Essais et des Principes: complémentarité matérielle tout d'abord puisque les éditions latines des Specimina physiques, bien plus fréquentes que les éditions françaises, furent couplées tout au long du $\mathrm{XVII}^{\mathrm{e}}$ siècle avec les Principia ${ }^{30}$; mais complémentarité conceptuelle tout aussi bien puisque les Principes, loin d'abolir le savoir des Essais, ne cessent d'y renvoyer comme à quelque chose d'acquis une fois pour toutes $^{31}$. Le véritable fondement de ce détour est cependant la thèse qui est soutenue dans un second temps : le statut des Essais et des Principes a beau ne pas être le même, les procédures à l'œuvre dans ces deux traités sont identiques - ce dont nous examinerons les conséquences.

28. Au comte d'Alais, 3 oct. 1642, in O.O. VI 156, trad. in B. Rоснот, Les Travaux de Gassendi sur Épicure et sur l'atomisme, 1619-1658, Paris, Vrin, 1944, p. 90.

29. $S P$, pars 1, lib. 2, cap. 5 : « Posse aliquam veritatem signo aliquo innoscere, et criterio dijudicari $»$, in 0.0. I 81-82.

30. Je dois cette remarque à Frédéric de Buzon, communication personnelle; pour plus de précisions, voir Albert-Jean GuIBERT, Bibliographie des auvres de Descartes publiées au XVII siècle, Paris, Éditions du CNRS, 1976.

31. Pour la Dioptrique, PP III 132, III 134, IV 189, IV 195, in A.T. IX-2, respectivement p. 183, 186, 310, 314. Pour les Météores, PP III 97, IV 19, IV 48-49, IV 87, IV 195, in A.T. IX-2, respectivement p. 158, 209, 227, 247, 314. 
Descartes entendait donner dans les Essais, non pas un exposé complet de sa physique, mais seulement des échantillons de sa méthode; il ne procédait pas a priori, c'est-à-dire en déduisant les effets de leurs causes, mais $a$ posteriori, c'est-à-dire en remontant des effets aux causes, tout en demandant à ses lecteurs de bien vouloir accepter initialement certaines suppositions correspondant en gros aux principes généraux qui peuvent être fondés métaphysiquement ${ }^{32}$. La distinction entre l'a priori et l'a posteriori est classique, et la confrontation de différents textes méthodologiques avait conduit au $\mathrm{XVI}^{\mathrm{e}}$ siècle certains commentateurs d'Aristote à élaborer la théorie du regressus demonstrativus : il s'agissait d'obtenir un savoir certain en combinant l'inférence d'un effet connu à une cause possible et l'inférence de cette cause possible à l'effet connu ${ }^{33}$. Descartes est proche de cette théorie lorsqu'il soutient que, dans les Essais, « les raisons s'entresuivent en telle sorte que, comme les dernières sont démontrées par les premières, qui sont leurs causes, ces premières le sont réciproquement par les dernières ». Il est vrai qu'il précise immédiatement :

« Et on ne doit pas imaginer que je commette en ceci la faute que les logiciens nomment un cercle; car l'expérience rendant la plupart de ces effets très certains, les causes dont je les déduis ne servent pas tant à les prouver qu'à les expliquer; mais, tout au contraire, ce sont elles qui sont prouvées par eux ${ }^{34}$. »

L'objet de ces précisions est de convaincre son lecteur qu'une démonstration a posteriori comporte suffisamment de certitude pour être appelée démonstration. Selon Descartes, une démonstration a posteriori serait à la fois une explication et une preuve; il ne s'agirait pas de deux opérations distinctes et successives, mais de la même opération considérée soit du point de vue des effets, soit du point de vue des causes. Étant de lui-même très certain, un fait n'aurait pas à être prouvé, mais à être expliqué, et son

32. DM, sixième partie, in A.T. VI, p. 75-76; Dioptrique, chap. l, in A.T. VI, p. 83; Météores, chap. I, in A.T. VI, p. 233; à Vatier, 22 fév. 1638, in A.T. I, p. 563; à Morin, 13 juil. 1638, in A.T. II, p. 200-201; passim.

33. John Herman RANDALL a attiré l'attention sur la théorie du regressus chez les aristotéliciens de la Renaissance in The School of Padua and the emergence of modern science, Padoue, Antenore, 1961. Nicholas JARDine fait le point sur cette théorie aussi bien que sur les critiques adressées à l'ouvrage de Randall dans son article, "Galileo's road to truth and the demonstrative regress ", Studies in the history and philosophy of science, vol. 7, 1976, p. 277 318. Pour une introduction synthétique aux problèmes épistémologiques de la Renaissance, voir N. JARDINE, "Epistemology of the sciences ", in The Cambridge history of Renaissance philosophy, éd. Charles B. Schmitt et Quentin SkInner, Cambridge, Cambridge University Press, 1988, p. 685-713.

34. $D M$, sixième partie, in A.T. VI, p. 76. Également à Morin, 13 juil. 1638, in A.T. II, p. 198. Sur la distinction entre a priori et a posteriori, voir les textes indiqués supra $\mathbf{n} .32$, ainsi que : à Mersenne, 10 mai 1632, in A.T. I, p. 250-252, cité supra n. 19 et à Plempius, 20 déc. 1637, in A.T. I, p. 476. 
explication consisterait à exhiber un processus causal qui conduit à sa production. Cette explication, considérée du point de vue des causes, serait une preuve : supposons les principes les plus généraux de la physique établis par la métaphysique, trouver la cause particulière d'un fait, c'est montrer qu'elle peut le produire. Mais on se heurte ici à une objection évidente : montrer qu'une cause particulière peut produire un effet, ce n'est pas montrer qu'elle est la seule à pouvoir le produire. À ceux des lecteurs qui lui adressaient cette objection, Descartes a donné principalement deux réponses.

En premier lieu, il affirmait que la conjonction de plusieurs inférences probables équivaut à une démonstration. Lorsque par exemple Libert Froidmont (dit Fromondus) s'étonne de certaines suppositions des Météorologiques (les parties de l'eau auraient la forme d'anguilles, celles de l'huile la forme de branches ${ }^{35}$ ), Descartes lui répond en construisant un ensemble de syllogismes de la forme : le rapport entre le comportement de l'eau et celui de l'huile dans telle circonstance est un indice (indicium) que l'eau est composée de corpuscules en forme d'anguilles et l'huile de corpuscules en forme de branches; or l'expérience montre que l'eau et l'huile se comportent ainsi dans cette circonstance; donc il est probable que l'eau est composée de corpuscules en forme d'anguilles et l'huile de corpuscules en forme de branches. Cet ensemble de syllogismes, considéré précisément comme un ensemble, a selon Descartes valeur de démonstration : «Bien que ces choses, étant considérées séparément, ne puissent constituer qu'une persuasion probable, lorsqu'elles sont toutes embrassées sous un même regard, elles sont démonstratives ${ }^{36}$. » Ce faisant, Descartes renvoie Fromondus à une procédure qui lui était familière. La rhétorique antique avait opposé les signes probables (indicia, $\tau \varepsilon \kappa \mu \varepsilon \rho \iota \alpha$ ) aux signes nécessaires (signa, $\sigma \eta \mu \varepsilon i \tilde{\alpha})$ : sans être démonstratifs pris un à un, les premiers emportent la conviction lorsqu'ils sont ajoutés les uns aux autres ${ }^{37}$. Dès le

35. Fromondus à Descartes, 13 sept. 1637, in A.T. I, p. 406-407. Pour tout dire, l'objection de Fromondus peut être comprise de deux manières. La première, que Descartes n'envisage pas, est la plus radicale : les principes généraux de la physique cartésienne seraient erronés, car l'eau et l'huile sont des corps homogènes au sens aristotélicien - au-delà d'un certain point, leur division est impossible ; en deça de ce point, le résultat de la division est toujours "de l'eau " et « de l'huile ». Descartes répond seulement à une version faible de l'objection de Fromondus : admettons qu'il y ait des parties de l'eau et de l'huile qui ne sont plus « de l'eau » et « de l'huile », mais des corpuscules doués d'une certaine figure, comment pouvonsnous connaître leurs figures?

36. Voir à Fromondus, 3 oct. 1637, in A.T. I, p. 422-423 : "Quae quamvis singula sejunctim considerata non nisi probabiliter persuadeantur, omnia tamen simul spectata demonstrant." "

37. QuiNTILIEN, in Institutio oratoria, V, 9, 3-5, définit les signes probables : " [...] quae etiam si ad tollendam dubitationem sola non sufficiant, tamen adjuncta caeteris, plurimum valent. " Les principaux passages fondant la théorie thétorique classique du signe se trouvent in Aristote, Rhétorique, I 2, 1357b et Premiers Analytiques, II 27 et Cić́ron, De inventione, 1, 48 ; Partitiones oratoriae, 10, 34 et Rhetorica ad Herennium, 2, 8, qui lui était alors attribuée. 
XIII ${ }^{e}$ siècle, la pensée juridique avait élaboré une théorie de l'addition des signes probables, des indices, des présomptions ou des degrés de preuve ${ }^{38}$. Aux $\mathrm{XVI}^{\mathrm{e}}$ et $\mathrm{XVII}^{\mathrm{c}}$ siècles enfin, la réhabilitation humaniste de la rhétorique et de la dialectique avait en particulier porté sur les raisonnements conjecturaux et les syllogismes probables ${ }^{39}$. Étant donné la fermeté avec laquelle Descartes avait condamné ce mode d'argumentation, source selon lui de tous les errements de la scolastique, il est pour le moins étonnant qu'il le réendosse ici, lui reprochant seulement d'être un peu fatigant pour les mains des typographes et les yeux des lecteurs ${ }^{40}$. On ne peut s'empêcher de se demander s'il ne se moque pas de Fromondus, parodiant une procédure que lui-même juge inapte à démontrer quoi que ce soit.

Descartes soulignait en second lieu qu'il ne se contentait pas de faire l'aller-retour entre une cause et un effet, mais allait de phénomènes connus à leurs causes, puis de celles-ci à des effets jusque-là inconnus. À JeanBaptiste Morin qui lui reprochait d'avoir commis un cercle dans la Dioptrique, il rappelle ainsi l'avertissement du premier chapitre : « [...] ce n'est pas un cercle de prouver une cause par plusieurs effets connus d'ailleurs, puis réciproquement de prouver quelques autres effets par cette cause ${ }^{41}$." Dans la Dioptrique, les "propriétés que l'expérience fait connaître " sont les suivantes : la lumière étend ses rayons partout en un instant, et en ligne droite; elle nous fait voir toutes sortes de couleurs; les rayons ne s'empêchent pas les uns les autres; ils se détournent ou perdent de leur force diversement selon les corps qu'ils rencontrent. Elles suggèrent trois comparaisons : la lumière est comme l'action transmise le long du bâton que tient un aveugle, comme la tendance du jus à s'écouler par des trous percés au fond d'une cuve pleine de raisin, et comme l'inclination à se mouvoir de balles, qui varie selon les corps qu'elles rencontrent ${ }^{42}$. Or non seulement ces trois comparaisons sont difficiles à concilier entre elles (c'est ce qui a souvent conduit les exégètes cartésiens à les analyser en

38. Sur l'arithmétique des degrés de preuve, voir Jean-Philippe LEvvy, "Le problème de la preuve dans les droits savants du Moyen Âge», in La Preuve. Deuxième partie. Moyen Âge et Temps modernes, Bruxelles, Éd. de la Librairie encyclopédique (Recueils de la société Jean Bodin pour l'histoire comparative des institutions, vol. XVII), 1965, en part. p. 148-160. L'importance de cette théorie juridique dans l'élaboration d'une notion quantitative du probable a été relevée par Lorraine DASTON, Classical Probability in the Enlightenment, Princeton, Princeton University Press, 1988, p. 38-46, puis par James FrankLIN, « SeventeenthCentury Probability », in The Uses of Antiquity. The Scientific Revolution and the classical tradition, éd. Stephen Gaukroger, Dortrecht, D. Reidel Publishing Company, 1991, p. 132139.

39. Je reviens dans la troisième partie de cet article sur cette réhabilitation.

40. Voir à Fromondus, 3 oct. 1637, in A.T. I, p. 424 : « Si talia omnia dialectico stilo deducere voluissem, immani profecto volumine typographorum manus et lectorum oculos fatigassem. »

41. À Morin, 13 juil. 1638, in A.T. II, p. 198.

42. Dioptrique, chap. 1, in A.T. VI, p. 83-92. 
termes de «modèles mécaniques »), mais Descartes les avait assimilées aux suppositions fausses des astronomes ${ }^{43}$. Comment pourrait-il donc soutenir sérieusement à Morin qu'elles constituent quelque chose comme une cause prouvée par des effets? Et ce d'autant plus que les « autres effets 》, les propriétés «qui ne peuvent être si aisément remarquées» sont la manière dont les corps se réfléchissent ou se réfractent, ou plus exactement la «réduction [des réflexions ou des réfractions] sous une même mesure ${ }^{44}$ », réduction qui n'est pas déductible des trois comparaisons. Là encore, la facilité avec laquelle Descartes adapte les procédures des Essais à la mode aristotélicienne est surprenante, et on supposera peut-être qu'il adopte ironiquement un langage suffisant à convaincre l'excellent aristotélicien qu'était Morin, tout en en connaissant parfaitement l'insuffisance démonstrative.

Ce qui rendait possible cette duplicité, c'est le statut particulier des Essais. Parce que Descartes ne prétendait y donner que des échantillons de sa méthode, il pouvait légitimement faire de son recours à des suppositions et à des preuves a posteriori un artifice provisoire de présentation, jouant de la différence entre ce qu'il choisissait de montrer au public et ce qu'il possédait par devers soi; et, lorsqu'un aristotélicien le sommait de s'expliquer sur la certitude de ses raisonnements, il se faisait une joie de lui montrer qu'ils étaient parfaitement conformes aux procédures classiquement employées dans l'École. Mais, en ce cas, quoi dans les Principes? À vrai dire, Descartes se sert toujours des mêmes procédures d'argumentations, il recourt toujours aux mêmes images, en bref sa pratique scientifique n'a pas changé ${ }^{45}$. Dans la mesure cependant où les Principes constituaient l'exposé complet et achevé de sa philosophie - mieux un «corps de philosophie »-, ces procédures et ces images ne pouvaient plus passer pour les effets secondaires du choix, délibéré mais provisoire, d'un certain mode d'exposé. Il devenait dès lors nécessaire de ménager dans la physique une place, pour ainsi dire officielle et permanente, aux hypothèses simplement probables. C'est précisément ce que Descartes effectue dans un des derniers articles des Principes:

«[...] comme un horloger industrieux peut faire deux montres qui marquent les heures en même façon, et entre lesquelles il n'y ait aucune différence en ce qui parait à l'extérieur, qui n'aient toutefois rien de semblable en la composition

43. Le texte est cité à l'appel de note 78. En réponse à une autre objection de Morin, Descartes, dans la lettre à Morin, 13 juil. 1638, in A.T. II, p. 198-199, précise que ses suppositions sont comparables seulement aux hypothèses astronomiques dont on peut tirer des conclusions vraies quoique elles-mêmes soient fausses ou incertaines.

44. Dioptrique, chap. II, in A.T. VI, p. 102.

45. On pourra s'exercer à vérifier cette permanence dans le cas du système du monde et de la lumière au livre III ou de l'aimant au livre IV des Principes de la philosophie. 
de leurs roues : ainsi il est certain que Dieu a une infinité de divers moyens, par chacun desquels il peut avoir fait que toutes les choses de ce monde paraissent telles que maintenant elles paraissent, sans qu'il soit possible à l'esprit humain de connaître lequel de tous ces moyens il a voulu employer à les faire. Ce que je ne fais aucune difficulté d'accorder. Et je croirais avoir assez fait, si les causes que j'ai expliquées sont telles que tous les effets qu'elles peuvent produire se trouvent semblables à ceux que nous voyons dans le monde, sans m'enquérir si c'est par elles ou par d'autres qu'ils sont produits. Mais je crois qu'il est aussi utile pour la vie, de connaître les causes ainsi imaginées, que si on avait la connaissance des vraies $[. . .]^{46}$. »

Les Principes ne s'arrêtent cependant pas à cet article. L'ultime tentative de Descartes pour surmonter la tension entre l'idéal d'une science qui ne laisse pas de place au doute et une physique pleine d'hypothèses est la notion de système que l'article suivant met en ouvre. Le moment du système est celui où la notion de cause certaine moralement succède à celle de cause possible, et l'analogie du chiffre à celle de la montre. On pense avoir déchiffré un chiffre non pas lorsqu'on sait avec certitude que tel terme correspond à tel terme, mais lorsqu'on a trouvé une interprétation d'ensemble qui tienne : il est peu probable qu'il existe deux interprétations cohérentes du même chiffre ${ }^{47}$. Au critère traditionnel de la vérité (l'adéquation singulière d'une proposition avec le réel) est dès lors substitué un indice par provision de la vérité (la cohérence intrinsèque d'un ensemble de propositions).

L'analogie du code, qu'on ne rencontre pas à ma connaissance chez Gassendi, sera reprise chez quantité d'auteurs à la suite de Descartes : il s'agit toujours de reconnaître le caractère seulement probable d'une proposition physique isolée tout en maintenant sa validité en tant qu'elle est articulée à d'autres propositions ${ }^{48}$. Plus généralement et pour finir, on peut dire que le

46. $P P$ IV 204, in A.T. IX-2, p. 322. Je reviens dans la seconde partie de cet article sur la signification de cette comparaison au Xvil ${ }^{e}$ siècle.

47. PP IV 205, in A.T. IX-2, p. 323-324 et in A.T. VIII-1, p. 327. Cette comparaison simplifie à l'évidence le problème : il est plus facile de déchiffrer un code alphabétique que d'expliquer les phénomènes naturels. Dans le premier cas, il s'agit seulement de recombiner des éléments en nombre fini et déjà connus; le nombre et la nature des éléments qui expliquent les phénomènes nous sont par contre initialement inconnus. Dans les Regulae, regula $\mathrm{x}$, in A.T. X, p. 404-405, le déchiffrement d'un code exemplifiait seulement la nécessité de procéder selon une méthode, fût-elle artificielle.

48. Jacques Rohault, Traité de physique, Paris, 1675 (cité par la suite comme TP), liv. I, chap. III, § 3, p. 22. LeIBNIz, à Conring, $19 / 29$ mars 1678, in Leibniz. Sämtliche Schriften und Briefe, éd. Preussische (puis Deutsche) Akademie der Wissenschaften, Darmstadt (puis Leipzig puis Berlin), 1923 sqq. (cité par la suite comme D.A.W.) II 2, p. 399, trad. in Leibniz. Euvres choisies, éd. Lucy Prenant, Paris, Aubier-Montaigne, 1972, (cité par la suite comme L.P.), p. 123. Christiaan Huygens, à Pierre Perrault, [1673], in CEuvres completes de Christiaan Huygens, éd. Société hollandaise des sciences, 22 vol., La Haye, 1888-1950 (cité par la suite comme S.H.S.), VII, p. 298. Robert BoYLE, The Excellency of the mechanical hypothesis, in The Works of the honourable Robert Boyle, éd. Thomas BırcH, 6 vol., Londres, 1772, 
destin épistémologique de la physique cartésienne dans la seconde moitié du xvir ${ }^{e}$ siècle se joue dans l'interprétation de ces deux articles. La physique cartésienne a été caractérisée à la fois comme hypothétique ${ }^{49}$ et comme systématique ${ }^{50}$, sans que ces deux interprétations soient considérées comme exclusives : le savoir n'aurait pas besoin d'être organisé en système si chaque proposition n'était pas hypothétique, et leur organisation en système ne suffit pas à effacer le caractère hypothétique de chaque proposition prise individuellement.

Que conclure au terme de cette première partie? Deux choses ont été distinguées chez Descartes : d'une part une théorie de la connaissance antisceptique et une théorie de la science rejetant le probable, d'autre part l'épistémologie hypothétique des Principes. Mais l'examen de l'œuvre de Descartes aussi bien que sa confrontation avec celle de Gassendi révèlent la pauvreté de l'expression "épistémologie hypothétique de la physique». Reconnaître qu'un auteur recourt à des hypothèses ne saurait être qu'un premier pas; il faut ensuite déterminer la fonction et la nature de ces hypothèses. C'est une analyse de ce genre, épistémologique au sens fort, qui a finalement été poursuivie. Nous avons montré pourquoi il y avait des hypo-

réimpr. Hildesheim, Georg Olms, 1966 (cité par la suite comme T.B.), IV, p. 77. Les variations de cette comparaison importent pour tout dire autant que sa récurrence : Rohault et Leibniz se contentent d'y faire allusion, alors que Huygens souligne que le déchiffrement d'un code gagne progressivement en certitude au fur et à mesure que de nouvelles occasions de le mettre à l'épreuve se présentent. Quant à Boyle, il se met dans la position d'un homme qui, ayant découvert le bon code, doit convaincre d'autres décodeurs que son code est bon et le leur faux : il ne saurait y parvenir par des arguments verbaux; seuls des tests expérimentaux pourront emporter la conviction de ses concurrents.

49. Voir Walter Charleton, Physiologia Epicuro-Gassendo-Charltoniana or a fabrik of science natural upon the hypothesis of atoms, Londres, 1654, réimpr. New York, Johnson Reprint Corporation, 1966 (cité par la suite comme EGC), p. 128 : arrêtant sa lecture des Principes à l'article IV 204, il en fait une justification définitive des opinions conjecturales, " which we desire may be candidly accepted in the latitude of probability only, or how it may be, rather than how it is ". Voir également Joseph GLanviLL, The Vanity of dogmatizing, (cité par la suite comme VD), chap. xxI, in Collected Works, Hildesheim, Georg Olms, 1970 (cité par la suite comme G.C.W.), I, p. 211-212, repr. in Scepsis Scientifica (cité par la suite comme SS), chap. XXv, in G.C.W. III, p. 155 : se référant au même article des Principes de la philosophie que Charleton, Glanvill soutient que « the grand secretary of nature, the miraculous Descartes [...] intends his principles but for hypotheses, and never pretends that things are really or necessarly, as he has supposed them ".

50. Voir par exemple les extraits du père Gabriel DanIEL, Le Voyage du monde de M. Descartes, cité in René Dugas, La Mécanique au xvIf siècle, Neuchâtel, Éditions du Griffon, 1956, p. 275, ceux du père René RapiN, Réflexions sur la philosophie ancienne et moderne et sur l'usage qu'on doit en faire pour la religion, « Réflexions sur la physique », $\$ \mathrm{X}$, Amsterdam, 1709, t. II, p. 401-402, cité in Gaston SortaIs, S.J., « Le cartésianisme chez les jésuites français au XvI' et au XVII' ${ }^{e}$ siècle ", Archives de philosophie, vol. VI, cah. 3, 1929, p. 5 et les remarques de C. Huygens, "Compte rendu de la Vie de M. Des Cartes par Baillet ", in S.H.S. X, p. 403-406; passim. Parmi les cartésiens, Régis est celui qui a le plus insisté sur le caractère systématique du savoir; voir par ex. Système de philosophie, préf. générale, op. cit. supra n. 3, t. I, n. p. et La Physique, avertissement, in ibid., p. 275-276. 
thèses dans les Principes: les principes généraux de la physique et les expériences disponibles ne suffisent pas à garantir l'univocité d'une chaîne déductive. Nous avons aussi soutenu que, si la pratique scientifique de Descartes était identique dans les Essais et dans les Principes, le statut du dernier de ces ouvrages l'avait obligé à reconnaitre aux hypothèses probables de la physique une place de droit, et non seulement de fait. Dans la seconde partie, cette perspective limitée est abandonnée et une thèse plus générale est examinée : la pratique des hypothèses aurait été liée à la conception de la physique propre à la philosophie mécanique.

$$
\text { II. — LES HYPOTHËSES ET LA PHILOSOPHIE MÉCANIQUE }{ }^{51}
$$

La multiplicité des auteurs analysés par Popkin n'empêche pas que son livre soit structuré de manière à dévoiler un enchaînement de causes et d'effets. Alors que protestants et catholiques s'affrontent sur la règle de la foi (chapitre I); les textes de Sextus sont de nouveau découverts (chapitre II); la fortune du scepticisme se profile sur fond de controverses religieuses, voire est conditionnée par elles :

« En contestant les critères traditionnels du savoir religieux, la Réforme avait posé une question fondamentale : comment justifier les fondements du savoir? Voilà qui allait déclencher une crise sceptique qui, loin de se confiner à la théologie, s'étendit bientôt au domaine des sciences et à l'ensemble du savoir humain ${ }^{52}$."

La crise sceptique est alors décrite comme un miasme qui se propage insensiblement de la sphère religieuse au domaine intellectuel en général, tant et si bien que certains finissent par soutenir qu'aucune certitude n'est possible dans les sciences (chapitres III à v). Cette position extrême suscite des contre-attaques successives (chapitres vi à $\mathrm{x}$ ); les deux demiers chapitres enfin montrent comment un tournant décisif s'accomplit chez Spinoza et Isaac La Peyrère : de fidéiste qu'il était, le scepticisme devient antireligieux. Quelle thèse fondamentale sur l'enchaînement des causes et des effets cette structure laisse-t-elle transparaître? Popkin veut-il dire que la religion a posé le problème, et que les sciences n'ont fait que le transposer, le refléter, ou le répercuter? Dénie-t-il aux sciences toute autonomie théo-

51. Sur les difficultés que présente la construction de la catégorie historique de philosophie mécanique, voir ma thèse de doctorat, La Philosophie mécanique (1630-1690), non publiée.

52. $H S$, p. 48. Cette thèse scande tout le livre : voir $H S$, p. 33, 92-93, 131, 156-157, passim. 
rique, car elles n'auraient ni la force de poser la question du savoir dans leurs propres termes, ni même celle d'infléchir la manière dont elle avait été posée dans la sphère religieuse?

C'est en tout cas la thèse que soutient Henry Van Leeuwen dans un ouvrage inspiré par Popkin. Selon lui, William Chillingworth et John Tillotson auraient tout d'abord trouvé des solutions au problème de la certitude dans un contexte religieux; dans un deuxième temps, ces solutions auraient été sécularisées par John Wilkins et Joseph Glanvill ; elles auraient ensuite été appliquées à la science par Robert Boyle et par Isaac Newton; en les thématisant philosophiquement, John Locke enfin tient lieu de chouette d'Athéna $^{53}$. La thèse de Popkin lui-même est plus nuancée : si la crise religieuse est le contexte historique indispensable pour comprendre l'acuité du renouveau sceptique, les différentes sphères théoriques gardent une certaine autonomie. Son ouvrage n'en induit pas moins l'idée qu'une crise sceptique déjà généralisée à l'ensemble du savoir est la raison principale pour laquelle des doutes étaient soulevés à l'égard des sciences; le copernicanisme ou le paracelsisme, par exemple, n'apparaissent pas comme des motifs qui déterminent la configuration spécifique des scepticismes propres au champ de l'astronomie ou au champ de la médecine, mais seulement comme des illustrations de la crise sceptique en général ${ }^{54}$. Corollairement, Mersenne et Gassendi semblent être les premiers à présenter certains énoncés physiques comme des hypothèses probables parce que, et seulement parce que, ils ont su modérer un scepticisme radical : le statut épistémologique de la physique serait principalement une conséquence de l'esprit philosophique du temps.

Tout en continuant à nous concentrer sur le domaine particulier du savoir que constitue la physique, nous défendrons dans cette deuxième partie une idée générale : on ne peut parler d'une crise du scepticisme sans étudier spécifiquement les différents champs du savoir concernés par cette crise; les arguments sceptiques n'auraient pas fait sens dans ces différents domaines si, chacun à leur manière, ils n'avaient pas été en crise, et la spécificité de chaque domaine a configuré l'utilisation de l'argumentation sceptique qui y était faite, autrement dit son scepticisme spécifique. Dans

53. Henry Van Leeuwen, The Problem of certainty in English thought. 1630-1690, $1^{\text {re }}$ éd. 1953, La Haye, Martinus Nijhoff, 1970, foreword, p. XIv. Cette thèse est réitérée aux charnières entre les différents chapitres : voir ibid., p. 48-49, 89-90, passim.

54. Par ex. $H S$, p. 89, 101, 105, 144, 146. Les travaux de $\mathrm{N}$. Jardine permettront de préciser cette question dans le cas de l'astronomie. 11 faudrait en faire autant dans le cas de la médecine étant donné d'une part le scepticisme de Galien et, d'autre part, le rôle de l'interprétation des signes dans la pratique médicale; on trouvera quelques indications dans W.P. D. WHIGHTMAN, « Les problèmes de méthode dans l'enseignement médical à Padoue et à Ferrare », in Sciences de la Renaissance, Actes du VII' congrès international de Tours, Paris, Vrin, 1973, p. 187 195, et dans Jean-Paul PItTion, «Scepticism and medicine in the Renaissance », in Scepticism from the Renaissance to the Enlightenment, op. cit. supra n. 2, p. 103-132. 
cette perspective générale, une question, laissée en suspens par Popkin, est tout d'abord traitée : quels savants sont touchés par la «crise sceptique »? La connivence entre l'ambition de la philosophie mécanique et le recours aux hypothèses est ensuite établie. Dans un troisième temps, nous dégageons la signification historique et conceptuelle du parallèle que font les philosophes mécaniques entre leurs hypothèses et celles des astronomes.

Popkin souligne à l'occasion la diversité des rapports entre science et scepticisme au XVII ${ }^{\mathrm{e}}$ siècle : non seulement « le scepticisme n'était [...] pas toujours du côté des bons ${ }^{55}{ }^{\prime}$, mais

«certains hommes de science d'un type nouveau (comme Galilée, Campanella, Descartes) [...] semblent [...] avoir cru que l'homme était capable de parvenir à la véritable connaissance du monde réel [...] Il n'y avait là aucun pyrrhonisme épistémologique, mais plutôt une sorte de réalisme ${ }^{56}{ }$.

On accordera aisément que la théorie de la connaissance qu'adopte un savant ne préjuge en général pas de ses réussites scientifiques; de même, les principes moraux ou esthétiques que met en place un philosophe ne sont pas corrélés directement avec sa moralité effective ou avec ses qualités d'artiste. En l'occurrence, l'argumentation sceptique a aussi bien servi que desservi la cause de la science. Elle a favorisé la science nouvelle dans ses luttes contre les sciences occultes ${ }^{57}$ et contre le dogmatisme scolastique ${ }^{58}$; de manière plus positive, elle a pu être utilisée pour imposer des idées nouvelles : puisqu'un phénomène prétendument manifeste comme la couleur est en réalité obscur, il n'y a aucune raison de refuser une idée prétendument obscure comme celle de gravitation universelle ${ }^{59}$. Inversement, elle a constitué un obstacle à la science lorsque, comme François de La Mothe Le Vayer, on juge inutiles ses peines et ses labeurs une fois établie qu'elle est principiellement impossible ${ }^{60}$, ou lorsque, comme Mersenne ou plus tard certains virtuosi de la Royal Society, on énumère les différentes hypothèses possibles pour expliquer un phénomène sans se donner les moyens de choisir la meilleure d'entre elles ${ }^{61}$. Il y a ici une idée générale que l'histoire du

55. $H S$, p. 130

56. $H S$, p. $200-201$.

57. $H S$, p. $128-130$.

58. Popkin le rappelle par exemple dans le cas de Sanchez (HS, p. 75-77), de Montaigne (HS, p. 89 et 93) ou de Gassendi (HS, p. 147-151); H. VAN LeEUwEN, op. cit. supra n. 53, dans le cas de Glanvill (p. 75-76) ou de Boyle (p. 94-96).

59. H. VAN LeEUWEN, op. cit. supra n. 53, p. 111-112, 136-138, passim.

60. HS, p. 130, 138-141. Une appréciation plus nuancée des positions de La Mothe Le Vayer se trouve in René PINTARD, Le Libertinage érudit dans la première moitié du xVIr siècle, Paris, 1943, réimpr. Genève/Paris, Slatkine, 1983, p. 143.

61. Zev BECHLER a souligné l'antagonisme entre le style dogmatique des lettres optiques de Newton et le scepticisme de bon ton à la Royal Society dans « Newton's 1672 optical contro- 
scepticisme a souvent inspirée : plus qu'une doctrine, le scepticisme est une manière d'argumenter; c'est pourquoi il est susceptible d'être utilisé à des fins opposées, qu'on soit dans le domaine de la science ou de la religion $^{62}$.

N'est-il cependant pas possible de dépasser le constat d'une variation sans raison? On pourrait être tenté de soutenir que le scepticisme des savants du XvII e siècle était inversement proportionnel à leur réussite scientifique : les ténors du scepticisme usuellement invoqués dans la littérature secondaire, qu'il s'agisse de Mersenne et Gassendi en France ou de Glanvill, Thomas Sprat et Boyle en Angleterre, ne sont pas des figures majeures de la «Révolution scientifique »; ses héros, Nicolas Copernic, Johannes Kepler, Galilée ou Newton, loin de douter de leur capacité à découvrir des propositions vraies, se sont considérés comme des prophètes chargés d'apporter au monde une vérité nouvelle ${ }^{63}$. Cette thèse historique aurait bien sûr admis des exceptions, et je pense ici à Christiaan Huygens; son principal défaut est cependant de dépendre de préjugés implicites sur la science : elle se réduirait à la mathématisation des phénomènes et son histoire serait une suite de coups de théâtre opérés par des théoriciens de génie. Aussi importe-t-il de distinguer différentes espèces de science ou différentes manières d'être un savant. L'existence de controverses sur l'exactitude d'une démonstration ou sur la voie la plus naturelle pour démontrer un théorème, voire de débats sur la certitude des mathématiques en général, n'empêchent pas les physiciens de considérer les objets mathématiques comme le modèle par excellence de vérités nécessaires et indubitables. Par extension, les énoncés physiques qui sont susceptibles d'être mis sous forme mathématique sont tenus pour certains; c'est en particulier le cas en mécanique et dans certaines parties de l'optique ou de l'astrono-

versies. A study in the grammar of scientific dissent $»$, in The Interaction between science and philosophy, éd. Yehuda Elkana, Atlantic Highlands, NJ, Humanities Press, 1974, p. 115-140.

62. Sur l'ambivalence de l'argumentation sceptique, voir $H S$, p. 27, 47, 238-239, passim, et C. B. Schmit, Cicero Scepticus. A study of the influence of the Academica in the Renaissance, La Haye, Martinus Nijhoff, 1972, p. 25. C. Larrère oppose le scepticisme comme tradition et le scepticisme comme argumentation dans la « Présentation » qu'elle a rédigée pour l'édition française, $H S$, p. 15-16. La distinction entre un scepticisme destructeur et un scepticisme constructif est un lieu commun de l'histoire du scepticisme; voir, par ex., T. GrEGORY, op. cit. supra n. 7, p. 21-22.

63. Benjamin NELSon file la métaphore du prophète aux p. 3-6 de son article, « The early modern revolution in science and philosophy ", in Boston studies in the philosophy of science, vol. 3, Dordrecht, D. Reidel Publishing Company, 1967, p. 1-40. Après avoir rappelé que les grands savants du Xvir ${ }^{\mathrm{e}}$ siècle se voyaient comme des prophètes, Nelson ajoute ironiquement, p. 12 : «[...] if we would believe Van Leeuwen and Popkin, it was not Galileo, Descartes, or even Bacon who initiated the philosophy or methods of modern science, but Chillingworth and Tillotson. " La comparaison du savant et du prophète n'a rien d'anachronique; selon une lettre de Chapelain à Vossius, 22 août 1663, in Lettres de Jean Chapelain, éd. Philippe TAMIzEY DE LARROQUE, Paris, 1880-1883, t. II, p. 326, n. 1, Descartes « traite sa physique en prophète, enveloppant d'ombrages sa doctrine et affirmant tout sans rien prouver ». 
mie. Toutefois, aussi importante qu'elle soit du point de vue de l'histoire sanctionnée, la physique mathématique n'est qu'un îlot dans l'océan des énoncés regroupés sous le nom de "philosophie naturelle » au XVII siècle; et cet océan comporte quantité d'hypothèses mal assurées. Les philosophes mécaniques en particulier présentent le plus souvent la manière dont ils expliquent les phénomènes et leurs changements comme des hypothèses; Larry Laudan a été jusqu'à soutenir que la «méthode des hypothèses » était une conséquence «logique » de la philosophie corpusculaire :

« [...] une fois la théorie corpusculaire de la matière acceptée, et avec elle la théorie de la connaissance qui fait les corpuscules inobservables par principe, il est tout à fait naturel d'admettre une version ou une autre de la méthode des hypothèses. En bref, la métaphysique et l'épistémologie de la philosophie mécanique conduisent, par leur propre logique interne, à défendre une certaine méthodologie ${ }^{64}$.»

La thèse de Laudan a ceci de stimulant qu'elle met en rapport l'ontologie des philosophes mécaniques et leur épistémologie; il reste cependant à éprouver sa validité historique. Laudan considère en effet que le raisonnement suivant va de soi : les philosophes mécaniques ne voyaient pas les corpuscules dont ils se servaient pour expliquer les phénomènes, donc ils devaient faire des hypothèses et justifier cette pratique inadmissible pour les aristotéliciens. Mais il ne procède pas à une démonstration systématique de cette thèse; son argumentation historique se réduit à deux points. En premier lieu, la «méthodologie » cartésienne serait « hypothétique », « probabiliste », ou « conjecturale» - ces adjectifs sont pour lui synonymes -; en second lieu, elle aurait exercé une influence décisive sur certains virtuosi anglais, Boyle, Glanvill ou Henry Power. L'emblème de la méthodologie de Descartes, mais aussi le chaînon décisif entre ce dernier et la Royal Society, serait le texte des Principes qui compare le physicien utilisant des hypothèses à un homme qui devrait deviner de l'extérieur le mécanisme d'une horloge ${ }^{65}$.

64. L. LAUDAN, op. cit. supra n. 9, p. 44. Laudan précise dans l'introduction ce qu'il entend par "méthodologie ", voir ibid, p. 2-4. Il distingue deux branches de la philosophie de la science : celle qui étudie les fondations conceptuelles d'une théorie scientifique spécifique, et celle qui cherche à comprendre selon quels principes différentes théories scientifiques sont comparées et validées; c'est la seconde qui est appelée « théorie des méthodologies scientifiques » et qui fait l'objet de son livre.

65. Ce texte est cité à l'appel de note 46. Sur l'image de la montre en général, voir Otto MAYR, Authority, liberty and automatic machinery in early modern Europe, Baltimore/ Londres, Johns Hopkins Press, 1986. Laudan spécifie que ce n'est pas cette image en général qui est propre à Descartes, mais son usage méthodologique; il retrouve ensuite un tel usage chez BoYLE, in The Usefulness of natural philosophy, in T.B. II, p. 45 et chez Glanvill, in $V D$, chap. XIX, in G.C.W. I, p. 180, repr. dans SS, chap. XIX, in G.C.W. III, p. 133. Dans ce dernier texte cependant, la comparaison d'une chose naturelle à une montre ne justifie pas le recours aux hypothèses, mais dénonce leur infériorité par rapport à un savoir qui résulterait 
Deux réserves factuelles peuvent être formulées à l'égard de cette argumentation. En premier lieu, qu'un auteur compare la connaissance des choses naturelles à l'identification d'un mécanisme horloger ne prouve pas qu'il soit sous l'emprise d'une « influence » cartésienne. En effet, Gassendi recourt lui aussi à cette comparaison pour souligner le caractère irrémédiablement hypothétique de notre savoir :

«Depuis bien longtemps, ce principe constitue pour moi une preuve que les natures des choses nous sont cachées : il appartient exclusivement à l'ouvrier de connaître la fabrication et la création de son œuvre; or nous, nous ne façonnons par notre travail aucune chose naturelle, ni pierre, ni plante, ni animal. À ce point que de même qu'un chien, quelque intelligent que soit par ailleurs cet animal, ne pourra pas savoir comment la machine d'une horloge, ou un autre ouvrage humain, est fait, puisque ce n'est pas un esprit canin qui a réalisé une œuvre de ce genre, de même un homme ne pourra pas bien savoir comment une plante naît, croît et produit des feuilles, des fleurs et des fruits qui sont toujours de son espèce parce que ce n'est pas un esprit humain qui a inventé une telle chose ${ }^{66}$.

En second lieu, Laudan associe le caractère hypothétique de la physique cartésienne à l'inobservabilité des corpuscules. Il est vrai que Descartes recourt à l'image de la montre pour expliquer « comment on peut parvenir à la connaissance des figures, grandeurs et mouvements des corps insensibles $^{67}$ ". Une telle explication n'a cependant pas sa nécessité propre dans l'exposé cartésien; elle intervient seulement à titre de réponse à une objection possible : «quaerent fortasse nonnulli », il y aura peut-être des gens pour venir me poser cette question. Plus généralement, la comparaison des choses naturelles à des montres ne s'inscrit pas seulement dans un contexte

d'une décomposition réelle des choses. C'est dire qu'il existe, non pas une, mais différentes interprétations épistémologiques de l'image de la montre (pour d'autres interprétations encore, voir Thomas Hobres, Le Citoyen, préf., trad. de Samuel Sorbière, rééd. de Simone GoyardFabre, Paris, Flammarion, 1982, p. 71; Glanvill, Plus ultra, in G.C.W. IV, p. 11 ; Boyle, The Christian Virtuoso, in T.B. VI, p. 721.722; LEIBNIZ, Specimen demonstrationum de natura rerum corporearum, [1], in D.A.W. VI 2, p. 300-301; LEIBNIZ, Summa hypotheseos physicae novae, [1], in D.A.W. VI 2, p. 327-328; LeIBniz, à Conring, début mai 1671, in D.A.W. II 1, p. 94.

66. À Herbert [cette lettre ne fut jamais envoyée], in O.O. III $413 \mathrm{~b}$. Également $R M$, contre la deuxième méditation, doute 8 , art. 2 , in 0.0 . III $312 \mathrm{~b}$ et in RochOT, 1962, p. 188; $S P$, pars 2 , sectio 1 , lib. 2, physicae proemium, in 0.0 . I 125b-126b. Ici comme ailleurs, il s'agit de situer le conflit entre Descartes et Gassendi plutôt que d'en nier l'existence. Il ne vient pas de ce que l'un use d'une comparaison, l'autre non, mais de ce que cette comparaison illustre : alors que Gassendi n'exclut pas que Dieu ait fait les choses d'une manière qui nous soit totalement incompréhensible, pour Descartes le seul problème est de choisir entre plusieurs explications possibles d'un même phénomène, étant entendu qu'aucune ne nous est principiellement incompréhensible.

67. PP IV 203, in A.T. IX-2, p. 321. 
épistémologique privilégiant la question des observables : si les hommes ne peuvent connaître avec certitude la structure microscopique des phénomènes, c'est tout aussi bien parce qu'ils ne peuvent pas les fabriquer ${ }^{68}$.

Outre ces réserves factuelles, l'argumentation de Laudan soulève un problème de droit : la notion d'une « logique interne » de la philosophie mécanique est un objet historiquement problématique. Même s'il y a eu une corrélation factuelle entre philosophe mécanique et recours aux hypothèses, cela ne signifie pas que les philosophes mécaniques aient déduit «logiquement » une certaine méthodologie de leurs convictions ontologiques; il est possible qu'à l'inverse la philosophie mécanique ait été admise en raison d'une modification des standards épistémologiques permettant de faire place aux hypothèses dans les sciences - c'est ce qu'on pourrait soutenir en s'inspirant de Popkin et de Van Leeuwen. Plus vraisemblablement encore, la composante ontologique et la composante épistémologique d'un tel complexe se sont rencontrées et renforcées mutuellement sans qu'aucune puisse être dite la cause de l'autre. Dès lors, il importe de se demander quel type d'argument historique on peut avancer pour montrer l'existence d'une corrélation entre philosophe mécanique et recours aux hypothèses. Deux choses sont possibles : d'une part, indiquer à titre heuristique ou spéculatif que certains complexes d'idées entretiennent des rapports de sympathie; d'autre part, exhiber des textes où certains philosophes mécaniques associent effectivement les entités constitutives de leur physique et le degré de certitude auquel celle-ci peut prétendre. Ces deux voies sont successivement empruntées dans les paragraphes suivants.

En évoquant prudemment les rapports de sympathie qui existent entre certains complexes d'idées, nous pensions opposer aristotéliciens et philosophes mécaniques. Pour les premiers, nos sens sont à la mesure des phénomènes, et pousser la décomposition des corps au-delà d'un certain degré n'a tout simplement pas de sens ${ }^{69}$. Pour les seconds, nos sens sont plutôt

68. On trouve l'idée que nous ne pouvons connaître les choses physiques parce que nous ne les avons pas créées in Marin MERSENNE, Questions physiques et mathématiques, question 22, "Quelles sont les vertus occultes? », éd. André Pessel, Paris, Fayard, 1985, p. 300; Gassend, $R M$, contre la deuxième méditation, doute 8 , art. 2 , in 0.0 . III 312 b et Rochot, 1962, p. 188; GasSENDI, SP, pars 2, sectio 1, lib. 2, physicae proemium, in O.O. I 125b-126b et ibid., pars 2, sectio 2, lib. 2, cap. 3 : «Quo sint positu, ordineve sita in universo sidera, quod quaeritur alias de ordine coelorum ", in 0.0 . I 559b-560a. Sur la signification et l'origine historique du slogan « on ne connaît rien sinon ce qu'on peut faire ou engendrer ", voir plus généralement T. Gregory, op. cit. supra n. 7, p. 70-77; Alistair C. CromBIE, Styles of scientific thinking in the European tradition. The history of argument and explanation especially in the mathematical and biomedical sciences and arts, Londres, Duckworth, 1994, vol. II, partie IV, chap. XII à XIV.

69. Keith Hutchison, «What happened to occult qualities in the Scientific Revolution », Isis, vol. 73, 267, 1982, en part. p. 238-240. Norma EMERTON, The Scientific Reinterpretation of form, Ithaca, Cornell University Press, 1984, en part. chap. II, p. 76-105. 
« dissimulateurs » que « trompeurs ${ }^{70}$, et nous devons chercher à dépasser les informations qu'ils nous livrent parce que la décomposition des corps va, soit à l'infini, soit du moins bien plus loin qu'ils ne peuvent l'indiquer $^{71}$. C'est ce qui explique le succès des observations microscopiques auprès des philosophes mécaniques. La philosophie mécanique ouvrait la possibilité théorique d'un regard microscopique: pour se servir d'un microscope, il faut être convaincu qu'il y a quelque chose à voir par-delà ce que nous voyons à l'œil nu; en retour, les observations microscopiques servirent non seulement à confirmer rétrospectivement certaines hypothèses mécaniques, mais aussi à légitimer par avance toutes les inférences à venir du sensible à l'insensible ${ }^{72}$.

Voici maintenant (c'est la seconde voie) deux textes liant explicitement l'ontologie de la philosophie mécanique et son recours aux hypothèses. Le premier est de Bernard Lamy :

« C'est autre chose de démontrer que les choses se peuvent faire comme on le dit, et qu'elles soient effectivement ce qu'on montre qu'elles pourraient être. [...] Le moyen de s'assurer de la vérité des hypothèses qu'on fait, [...] c'est de tâcher de voir par les yeux du corps ce qu'on n'apercevait que par des conjectures en raisonnant. Les télescopes et les microscopes servent à cela [...] [Mais] il faut reconnaître qu'en une infinité de choses, avec tous les secours du micro-

70. La formule est de LeIBNIz dans sa lettre à Oldenburg, 28 sept. 1670, in D.A.W. II 1, p. 63 : "Sensus nostros numquam mendaces, plerumque tamen dissimulatores esse. " Pour l'idée, voir Michel DE MonTaIGne, Essais, liv. II, chap. XII, éd. Albert ThIBaudet, Paris, NRF, 1940, p. 573-578; Gallé́, à Dini, 21 mai 1611, in Galilée. Dialogues et lettres choisies, trad. de Pierre-Henri Michel et Giorgio de Santillana, Paris, Hermann, 1966, p. 377 ; Descartes, $P P$ IV 201, in A.T. VIII-1, p. 324; GASSENDI, DP, liv, second, sixième dissertation, art. 5 , in O.O. III 203a et in Rochot, 1959, p. 484; RoBERVAL, fragment cité in R. LeNoble, op. cit. supra n. 7, p. 385; MariotTe, Essai de logique, première partie, principe 30 et seconde partie, deuxième discours, article II, in éd. Guy PICOLET et Alan GABBEY, Paris, Fayard, 1992 (cité par la suite comme $E L$ ), p. 24, 94 ; Charleton, EGC, p. 113-114; Boyle, The Skeptical Chemist, in T.B. I, p. 516; Glanvill, VD, chap. 1, in G.C.W. I, p. 5-7, passim.

71. GASSENDI, $S P$, pars 2 , sectio 1, liber 3, cap. $6:$ « De ipsis atomorum proprietatibus $»$, in O.O. I 269a; Charleton, EGC, p. 116; Robert Hooke, Micrographia, préf., n. p., Londres, 1665, réimpr. Lincolwood, Science Heritage Ltd, 1987; Thomas SPRAT, History of the Royal Society, Saint Louis/Londres, Washington University Press/Routledge and Kegan Paul Ltd, 1958 (cité par la suite comme HRS), p. 384; Gérauld DE CoRdemoY, Six discours sur la distinction du corps et de l'âme, troisième discours, in CEuvres complètes, éd. Pierre ClaIR et François GIRBal, Paris, Presses universitaires de France, 1968, p. 125-126; J. Rohault, TP, liv. I, chap. XXI, $\$ 2$, t. 1 , p. 160-161.

72. Sur l'interaction entre microscopie et la philosophie mécanique (atomisme ou corpuscularisme) au XVII siècle, voir Christoph MEINEL, "Early seventeenth-century atomism ", Isis, 79, 1988, en part. p. 81-84 ; C. MEINEL, « Das letzte Blatt im Buch der Natur. Die Wirklichkeit der Atome und die Antinomie der Anschauung in den Korpuskulartheorien der frühen Neuzeit », Studia Leibnitiana, XX/1, 1988, en part. p. 8-15; Catherine WILson, The Invisible World, Princeton, Princeton University Press, 1995, en part. p. 29-69; Christoph LUTHY, "Atomism, Lynceus, and the fate of seventeenth-century microscopy ", Early Science and medecine, vol. 2, 1, 1996, p. 1-27; passim. 
scope, des machines pneumatiques, de la chimie, nous ne pouvons pénétrer ce que la nature nous a voulu cacher. Nous ne voyons pas ce qu'elle est dans l'intérieur. Que peut donc faire un physicien, que de conjecturer ${ }^{73}$ ?"

Le second est de Pierre-Sylvain Régis. Ce dernier appelle «physique spéculative » ou « problématique » la partie de la physique qui est amenée à faire des conjectures parce qu'elle traite d'entités insensibles. Après avoir distingué le corps mathématique, le corps mécanique et le corps physique, il remarque que leurs propriétés ne sont pas étudiées de la même manière :

«Les propriétés du corps mathématique se déduisent aisément de sa nature, parce qu'elle est très simple et très aisée à comprendre [...] On explique aussi facilement les effets des machines, parce que leurs ressorts étant grossiers et palpables, on peut aisément apercevoir les rapports qu'ils ont entre eux, et prévoir ainsi l'effet qu'ils doivent produire, lorsqu'ils agissent tous ensemble. Mais il n'en est pas de même du corps physique, comme ses parties sont insensibles, on n'en peut apercevoir ni l'ordre ni l'arrangement, et tout le mieux qu'on saurait faire, c'est de les deviner de ces effets. Il y a donc deux parties dans la physique [...] : la partie pratique de la physique consiste dans l'observation exacte de tous les effets que chaque corps physique peut produire; et la partie spéculative consiste dans les raisonnements qu'on peut faire pour découvrir les causes de ces effets [...] La physique spéculative ne [peut] se traiter que de manière problématique, et tout ce qui est démonstratif ne lui [appartient] pas $^{74}$. 》

Il ne saurait être question de dissimuler les réticences que peut inspirer à l'historien l'idée qu'une certaine méthodologie découle «logiquement » d'une ontologie - rien n'est, d'une certaine manière, plus imprévisible que le destin des idées. Les quelques éléments dont nous disposons semblent cependant indiquer qu'il a bien existé une collusion historique entre la philosophie mécanique et le recours aux hypothèses. En ce sens, l'introduction du vocabulaire des hypothèses dans le champ de la physique doit être analysée non seulement comme une conséquence d'une crise sceptique générale, mais en fonction de la manière de concevoir la tâche du physicien propre à la philosophie mécanique. Nous aimerions pour finir prolonger une autre suggestion de Laudan. D'après lui, en effet, les convictions ontologiques des physiciens non seulement induisent chez eux certaines méthodologies, mais les amènent à élaborer, lorsqu'elles vont à

73. Bernard Lamy, Discours sur la philosophie, in Entretiens sur les sciences, éd. Pierre Clair et François Girbal, Paris, Presses universitaires de France, 1966, p. 257-259.

74. P.-S. Regis, op. cit. supra n. 3, La Physique, Avertissement, t. I, p. 274-275. Dans la tradition épicurienne, l'adjectif "problématique " désigne les cas où plusieurs explications d'un même phénomène sont possibles: voir par ex. $S P$, pars 2 , sectio 1 , liber 4 , cap. 1 : «Cujusmodi sint quae a physicis requiruntur causae ", in O.O. I 286b, passim. 
l'encontre des normes admises, des arguments pour les défendre. Comment donc les philosophes mécaniques justifiaient-ils leur recours à des hypothèses en philosophie naturelle?

À l'évidence, tous les moyens sont bons. Parfois, ils invoquent l'autorité d'un texte d'Aristote : «Pour les phénomènes qui échappent à nos sens, nous estimons en avoir donné une explication satisfaisante pour la raison quand nous les avons ramenés à des faits possibles ${ }^{75}$. 》 D'autres fois, ils présentent la manière dont ils inferrent la structure insensible d'un phénomène à partir de sa structure sensible comme un déchiffrement de signes ${ }^{76}$. Parfois encore, ils comparent l'usage d'hypothèses en philosophie naturelle à la procédure arithmétique appelée regula $\mathrm{falsi}^{77}$. La justification la plus fréquente me semble cependant être la comparaison qu'ils font entre leur pratique et celle des astronomes qui forgent des hypothèses. Ainsi Descartes affirme-t-il pouvoir se dispenser d'exposer la nature de la lumière en recourant à des comparaisons analogues aux «suppositions » des astronomes :

«Il suffira que je me serve de deux ou trois comparaisons [...]; imitant en ceci les astronomes, qui, bien que leurs suppositions soient presque toutes fausses ou incertaines, toutefois, à cause qu'elles se rapportent à diverses observations qu'ils ont faites, ne laissent d'en tirer plusieurs conséquences très vraies et très assurées ${ }^{78}$. "

Tant qu'on en reste au niveau des métaphores, l'opposition traditionnelle entre Descartes et Gassendi ne résiste pas à l'épreuve des textes. Gassendi

75. ARISTote, Météorologiques, I 7, 344a 5-7, éd. et trad. par Pierre Louss, Paris, Belles Lettres, 1982. Ce passage était déjà cité par Nifo (Augustini Niphi Philosophi Suessani), Expositio [...] de physico auditu, Venise, 1552, cité in J. H. Randall, op. cit. supra n. 33, p. 45-46. Au XVII siècle, il l'est par GASSENDI, $D P$, livre premier, cinquième dissertation, art. 9, in O.O. III 130a et in ROCHOT, 1959, p. 134; DesCARTES, PP IV 204, in A.T. VIII-1, p. 327 et IX-2, p. 323; Charleton, EGC, p. 128; Boyle, The Usefulness of natural philosophy, in T.B. II, p. 45; passim.

76. Ainsi Descartes dans le texte cité et commenté à l'appel de note 36. GasSENDI, SP, pars 1, lib. 2, cap. $5:$ "Posse aliquam veritatem signo aliquo innoscere, et criterio dijudicari », in O.O. I 81a, défend, contre les sceptiques, l'idée qu'il est possible de connaître avec certitude les choses de la nature en interprétant certains signes naturellement cachés. MARIOTTE, EL première partie, principes 44 et 52 , respectivement p. 28 et 34 , lie les propositions vraisemblables à l'interprétation des signes. Passim.

77. BovLE, Some considerations touching experimental essays in general, in T.B. I, p. 303; HоOKE, Of comets and gravity, in The Posthumous Works, Londres, 1705, New York/Londres, Johnson Reprint Corporation, 1969, p. 173.

78. Dioptrique, chap. I, in A.T. VI, p. 83. Dans la Dioptrique et dans la correspondance avec Morin, Descartes emploie indifféremment les termes « supposition » et « hypothèse ». Il invoque la pratique des astronomes dans d'autres contextes, qu'il assume des postulats qui ne sont pas reçus par tous (Regulae ad directionem ingenii, regula XII, in A.T. X, p. 417) ou qu'il recoure à une supposition aussi fausse que celle d'un Dieu trompeur (Responsio ad quintas objectiones, in A.T. VII, p. 349-350). 
compare lui aussi ses hypothèses atomiques aux «fictions » des astronomes :

«Je me sers [de ces fictions] comme d'hypothèses par lesquelles je m'efforce, autant que je le peux, de comprendre et d'expliquer l'industrie admirable avec laquelle l'ouvrier souverainement sage a voulu que ses propres cuvres manifestent ses actions. Et cela de la même façon que les astronomes lorsqu'ils introduisent dans le ciel des hypothèses, ou plutôt des fictions ${ }^{79}$. »

Nous nous sommes contentés de citer des textes de Descartes et de Gassendi, mais la référence aux hypothèses incertaines, voire fausses, des astronomes est banale dans les textes du Xvil ${ }^{\mathrm{e}}$ siècle qui soulignent la nécessité d'hypothèses en physique ${ }^{80}$, comme d'ailleurs dans le domaine moral ou politique ${ }^{81}$. La première question est donc de savoir dans quelle mesure le terme " hypothèse » faisait autorité en astronomie et pour quelles raisons; pour y répondre, nous nous appuierons ici principalement sur les travaux de Nicholas Jardine ${ }^{82}$. Nous nous demanderons ensuite ce qui justifiait et ce qui limitait la référence à l'astronomie que faisaient les philosophes mécaniques.

Si les astronomes avaient depuis longtemps remarqué qu'il peut y avoir plusieurs manières de sauver les phénomènes ${ }^{83}$, c'est dans la seconde moi-

79. De proportione, epistola III, in O.O. IV 635a.

80. Isaac BeEcKman, 19 oct.-[2] nov. 1619, in Journal tenu par I. Beeckman de 1604 à 1634, éd. Cornelis De WaARD, La Haye, Martinus Nijhoff, 1939-1953, t. I, p. 344; Blaise PAsCal, au père Noël, 29 oct. 1647, in Euvres complètes, éd. Jacques Chevalier, Paris, NRF, 1954, p. 375; MaRIOTTE, $E L$, première partie, principe 52 et seconde partie, deuxième discours, article II, respectivement p. 34 et 96; Tommaso CoRnelo, Progymnasta physica, Venise, 1663, p. 30, cité in Della scienza mirabile alla scienza nuova. Napoli e Cartesio, Naples, Biblioteca nazionale di Napoli et Istituto italiano per gli studi philosophica, 1997, p. 30; Luca Tozzl, Appendix ad commentarium, Aphorismi III, liber I, in Opera omnia medica, Venise, 1740, II, p. 6, cité in ibid., p. 45. Plus généralement, de nombreux concepts astronomiques sont importés dans la physique au XvII siècle, par ex. " théorie », " phénomène », « système ».

81. Voir François de LA MOTHE Le VAYER, Dialogues faits à l'imitation des Anciens, 16301631 , éd. André Pessel, Paris, Fayard, 1988, p. 330-331; Marin Cureau de La Chambre, Système de l'âme, Paris, 1664, préf., cité par R. SAsso, "Système et discours philosophique au xvI" siècle ", Recherches sur le XVI" siècle, 2, p. 127.

82. Trois publications sont en jeu : "The forging of modern realism. Clavius and Kepler against the sceptics ", Studies in the history and philosophy of science, vol. 10, 2, 1979, p. 141-173; The Birth of history and philosophy of science. Kepler's A Defense of Tycho against Ursus with essays on its provenance and significance, Cambridge, Cambridge University Press, 1984; «Scepticism in Renaissance astronomy. A preliminary study ", in Scepticism from the Renaissance to the Enlightenment, op. cit. supra n. 2, p. 83-102. Voir également Edward Grant, Planets, stars, and orbs. The medieval cosmos, 1200-1687, Cambridge, Cambridge University Press, 1994; Michel-Pierre Lerner, Le Monde des sphères, vol. 1, Paris, Belles Lettres, 1996.

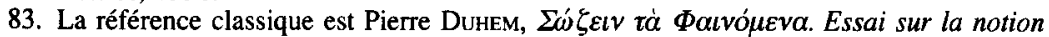
de théorie physique de Platon à Galilée, Paris, Hermann, 1908. Geoffrey E. R. LLOYD, "Saving the appearances », Classical Quarterly, 28, 1978, p. 202-222, et N. JARDINE, "The forging of modern realism », p. 141-173, repr. avec quelques modifications in The Birth of history and philosophy of science, op. cit. supra n. 82, p. 225-239, ont cependant montré que 
tié du Xvi siècle que l'idée que les propositions astronomiques sont seulement des hypothèses probables était devenu un lieu commun. Selon Jardine, cette idée était parfois associée à une épistémologie sceptique au sens propre du terme : les positions sur les hypothèses astronomiques de Philipp Schwarzerde (dit Melanchton), d'Andreas Osiander et d'Erasmus Reinhold auraient été liées à l'augustinisme modérément sceptique de certaines universités allemandes; les positions de Nicolai Reyners Baer (dit Ursus) rappelleraient le scepticisme polémique d'Agrippa von Nettesheim ${ }^{84}$. Plus généralement cependant, ce lieu commun dépend de l'état du savoir astronomique, et nous nous permettrons de simplifier une histoire complexe, notre objet n'étant pas les épistémologies astronomiques, mais leur exploitation par la philosophie mécanique. En gros, le conflit entre les deux théories existantes (épicycles et excentriques ptoléméens, d'une part, et sphères concentriques de la cosmologie aristotélicienne, d'autre part) avait été géré successivement de deux manières. À l'imitation d'Alhazen, les astronomes cherchèrent tout d'abord à élaborer des modèles physiques permettant un compromis entre ces deux théories. Vers la fin du Xvi siècle, la plupart des astronomes préféraient argumenter en termes de répartition des tâches intellectuelles : aux naturales d'enquêter sur la nature même des choses célestes; aux mathematici de suggérer des hypothèses permettant de sauver les phénomènes même si elles n'ont aucune charge réelle ${ }^{85}$.

Pas plus que les différentes sciences, les différentes conceptions de la science ne marchent au même pas - parfois même, elles procèdent par chassés-croisés : alors que Christoph Clavius, Kepler ou Galilée réagissent, au nom de la physique, contre la tendance à parler d'hypothèses probables en astronomie, les philosophes mécaniques se réclament de la pratique astronomique pour justifier l'usage d'hypothèses en physique. Ils estimaient se trouver dans une position analogue à celle des astronomes : de même que notre situation d'animaux terrestres nous interdit tout choix direct entre les différentes hypothèses astronomiques, de même notre position sur l'échelle des êtres, qu'il s'agisse de notre constitution sensorielle ou de nos capacités déductives, ne nous révèle pas directement la structure microscopique des corps. Aussi la concurrence des différents systèmes, en particulier celui de Descartes et celui de Gassendi, et l'existence, à l'intérieur d'un système donné, de différentes explications possibles d'un même phénomène sont souvent comparées à la rivalité entre les trois systèmes du monde. À l'instru-

que les partis pris théoriques de Duhem l'avaient conduit à rallier sous la bannière du réalisme des positions épistémologiques bien distinctes.

84. Respectivement N. JARDINE, « The forging of modern realism », p. 146-153 et ID., The Birth of history and philosophy of science, p. 240 , op. cit. supra n. 82 .

85. N. JARDINE, «The forging of modern realism », art. cit. supra n. 82, p. 229-230, 238243; M.-P. LeRNER, op. cit. supra n. 82, p. 111-130. 
ment emblématique de l'astronomie qu'était le télescope, les philosophes mécaniques faisaient correspondre le microscope : l'un et l'autre auraient dû nous permettre de savoir ce qu'étaient réellement les phénomènes ${ }^{86}$.

L'analogie entre hypothèses mécaniques et astronomiques est cependant loin d'être parfaite : les premières sont plus risquées que les secondes. Elles ont, en effet, pour objectif d'expliquer les qualités sensibles par des structures géométriques en mouvement et mettent à cet effet en rapport deux termes incommensurables: même si l'on pouvait établir que tel mouvement produit telle couleur, on ne comprendrait pas pour autant le rapport entre ce mouvement et cette couleur ${ }^{87}$. Les hypothèses astronomiques au contraire vont du même au même puisqu'elles consistent à supposer que les planètes ont tel ou tel mouvement réel pour rendre compte de leur mouvement apparent. C'est dire que la référence à l'astronomie ne constituait pas un modèle épistémologique au sens fort du terme; il s'agissait plutôt de parer des propositions mal assurées, et par conséquent attaquables par les aristotéliciens qui prétendaient posséder une science certaine, du prestige de la science mixte déjà bien établie qu'était l'astronomie. En ce sens, on peut supposer (et il y a là une conjecture historique à vérifier systématiquement) que c'est en raison de ce prestige que les philosophes mécaniques se sont référés si souvent aux hypothèses astronomiques.

Alors que la première partie était centrée sur un corpus de textes limité - l'œuvre d'une individualité appelée Descartes --, la deuxième partie a changé de perspective, cherchant à caractériser les savants qui recouraient à des hypothèses en physique. Nous avons en ce sens cherché à préciser historiquement la thèse, avancée par Laudan, qu'il existait un lien entre méthodologie hypothétique et philosophie mécanique. Pour finir, nous avons montré que la comparaison que les philosophes mécaniques faisaient entre leurs hypothèses et celles des astronomes, tout en ayant un fondement historique, procédait sans doute du désir de légitimer une pratique incer-

86. Francis Bacon, Novum Organum, II 39, trad. de Michel Malherbe et Jean-Marie PousSEuR, Paris, Presses universitaires de France, 1986, p. 270-271; GASSENDI, SP, pars 1, lib. 2, cap. 5 : « Posse aliquam veritatem signo aliquo innoscere, et criterio dijudicari », in 0.0 . I 82a; Henry Power, Experimental Philosophy, préf., Londres, 1666, repr. New York, Johnson Reprint Corporation, 1966, n. p.; Hooke, Micrographia, op. cit. supra n. 71, préf., n. p.; Glanvill, Plus Ultra, chap. vil, in G.C.W. IV, p. 51-57. C. Lüthy, art. cit. supra n. 72, p. 1-6, examine ce qui motive et ce qui limite l'analogie entre ces deux instruments.

87. Gassend, De apparente magnitudine, in O.O. III 472 a; Charleton, EGC, p. 197; BoYLe, The Excellency of theology, in T.B. IV, p. 44; BoYLE, Of the positive or privative nature of cold, in T.B. III, p. 740; BoYLE, The Experimental History of colours, in T.B. I, p. 696; LeIBNIz, Monadologie, \& 17, in L.P., p. 398; LockE, Essai philosophique concernant l'entendement humain, IV $3 \S 8$, trad. de Pierre Coste, Amsterdam/Paris, 1755, réimpr. Paris, Vrin, 1983, p. 461-462. L'incommensurabilité du rapport entre la sensation et son explication mécanique a été thématisée sous le nom d'irrationnel par Émile MEYERSON, in Identité et réalité, Paris, Vrin, 1951, p. 334 sqq. 
taine. Dans la troisième et dernière partie de cet article, une tout autre perspective est choisie: il s'agira de savoir dans quelle mesure on peut comprendre le recours aux hypothèses, en fonction non plus des contenus ontologiques de la physique, mais des règles de communication du savoir qui apparaissent dans les premières institutions scientifiques.

\section{III. — LES HYPOTHÈSES DANS UN CONTEXTE INSTITUTIONNEL}

Selon l'Histoire du scepticisme, le scepticisme modéré aurait été d'une importance décisive pour la pensée moderne; il aurait, en effet, déterminé l'attitude scientifique moderne, antimétaphysique, positiviste, pragmatiste, empiriste ${ }^{88}$. Cet impact fut différé conformément à un schème classique en histoire, celui de la révolution manquée ou de la répétition générale :

«Même si les formulations les plus théoriques de ce scepticisme modéré ou constructif virent probablement le jour au début du xvıl ${ }^{\mathfrak{e}}$ siècle, il fallait encore qu'un nouveau dogmatisme se développât et fût renversé avant que cette nouvelle solution à la "crise pyrrhonienne" pût être acceptée. Il fallut attendre que David Hume présentât cette position et que Mill et Comte l'interprétassent au $\mathrm{XIX}^{\mathrm{e}}$ siècle pour qu'elle fût enfin respectable d'un point de vue philosophique ${ }^{89}$. »

Dans la préface qu'il rédige pour le livre de Van Leeuwen une dizaine d'années après, plutôt que de repousser le triomphe du scepticisme dans le temps, Popkin le délocalise géographiquement; le schème est alors celui du relais des nations : la flamme sceptique s'éteint sur le continent lorsqu'elle embrase l'Angleterre ${ }^{90}$. Il y a là la version faible d'une thèse aujourd'hui assez répandue : le « scepticisme », le «probabilisme », le « faillibilisme », le «phénoménisme » ou le «pragmatisme » serait au XvII siècle caractéristique de la Science anglaise; quant au continent, il serait abandonné au despotisme d'un cartésianisme dogmatique ${ }^{91}$. Dans cette troisième et dernière partie, nous commençons par rappeler brièvement l'état de la question.

88. $H S$, p. 151, 179, 191, 197, 199. Pour une différenciation de ces catégories, voir par ex. Ian Hacking, Concevoir et expérimenter, trad. de Bernard DuCrest, Paris, Bourgois, 1989, en part. chap. III et IV.

89. $H S$, p. 179. Également $H S$, p. 202.

90. Voir H. VAN LEEUWEN, op. cit. supra $\mathrm{n}$. 53, foreword, p. IX : «The constructive scepticism of Sanchez, Mersenne and Gassendi led to a theoretical empirism and positivism that was probably too complex for the state of science of their day. On the other hand, it led to little of importance in experimental and practical results, and it was overshadowed by the dogmatic metaphyscial theory of their great contemporary, René Descartes. [...] But a new version of constructive scepticism was developping in England, among the theologians and scientists of the Royal Society, and with the scientific success of the Society, its theoretical outlook also triumphed. » B. NELSON, art. cit. supra n. 63, p. 11-12, relève lui aussi ce passage.

91. H. VAN LeEUwEN, op. cit. supra n. 53, p. 4 et p. 12, n. 30, fait l'impasse sur le scepticisme continental, à deux exceptions près. Barbara SHAPIRO, in Probability and certainty in 
Nous examinons ensuite les problèmes historiques et les limites conceptuelles de l'idée selon laquelle le scepticisme expérimentaliste en vogue en France et en Angleterre à la fin du $\mathrm{XVII}^{\mathrm{e}}$ siècle serait un discours déterminé par les exigences d'une " civilité savante».

Bien des historiens ont remarqué que les virtuosi de la Royal Society mirent les « faits » à l'honneur : ils en font des évidences premières, analogues aux axiomes mathématiques ${ }^{92}$. Mais, alors que des théorèmes peuvent être déduits des axiomes mathématiques, aucune proposition ne peut être dérivée déductivement d'un fait singulier; conséquemment, Boyle, Glanvill, Hooke ou Sprat, par exemple, appellent « hypothèse » tout ce qui n'est pas «fait », aussi bien une théorie scientifique qu'un système métaphysique, l'explication d'un phénomène particulier que l'interprétation d'un événement passé. Toujours pour ces virtuosi, la distinction du fait et de l'hypothèse est une affaire de morale aussi bien que de science : non seulement le dogmatique s'abuse intellectuellement en ne distinguant pas le vrai du probable, le fait de l'hypothèse, mais il est moralement coupable car il manque de modestie. Aussi est-il selon eux «convenable » que chaque savant présente ses théories comme des conjectures probables et évite de contredire frontalement les autres savants, du moins tant que ceux-là respectent eux aussi les règles du jeu ${ }^{93}$. Ce discours officiel n'était pas un idéal sans effectivité : la Royal Society censurait ceux qui s'en écartaient. Ainsi la lettre que Newton avait adressée en février 1672 à Henry Oldenburg fut-elle publiée dans les Philosophical Transactions seulement après que ce dernier eut supprimé le passage suivant, jugé trop dogmatique :

«Un philosophe naturel ne s'attendrait pas à voir la science de celles-ci [les couleurs] devenir mathématique, et pourtant j'ose affirmer qu'elle contient autant de certitude que toute autre partie de l'optique. Car ce que je dirai d'elles n'est pas une hypothèse mais la plus rigide des conséquences, ce n'est pas conjecturé selon cette pauvre inférence "c'est ainsi parce ce n'est pas autrement, ou parce que cela satisfait tous les phénomènes" (la rengaine uni-

seventeenth-century England, Princeton, Princeton University Press, 1983, introd., p. 4, 12, 14, caractérise d'entrée de jeu la science anglaise par son probabilisme.

92. La base historique et historiographique du paragraphe synthétique qui suit est constituée par les textes de Hooke, Boyle, Glanvill ou Sprat qu'on trouvera, par ex., in L. LAUDAN, op. cit. supra n. 9, p. 44, p. 27-58; H. VAN LeEUWEN, op. cit. supra n. 53, p. 71-105; Z. BECHLER, op. cit. supra n. 61, p. 115-140; B. SHAPIRo, op. cit. supra n. 91, p. 44-56; Simon SCHAFFER, "Making certain ", Social Studies of sciences, vol. 14, 1983, p. 137-152; Steve Shapin et Simon ScHAFrer, Léviathan et la pompe à air. Hobbes et Boyle entre science et politique, trad. de Thierry Pí́lat, Paris, La Découverte, 1993. Sur la spécificité et l'origine des faits baconiens, voir L. DASTON, «Baconian facts, academic civility, and the prehistory of objectivity », in Rethinking objectivity, éd. Alan MEGllL, Durham/Londres, Duke University Press, 1994, p. 37-58; B. ShapIRo, « The concept "fact". Legal origins and cultural diffusion », Albion, vol. 26, 2, 1994, p. 227-252.

93. BoyLE, Some Considerations touching experimental essays, in T.B. I, p. 311-312; Glanvil, SS, chap. XXVII, in G.C.W. III, p. 169-171; SPRAT, HRS, p. 32-34; passim. 
verselle des philosophes) mais prouvé par l'intermédiaire d'expériences permettant des conclusions directes et qui ne laissent pas l'ombre d'un doute ${ }^{94}$. »

Faire l'apologie du fait et appeler « hypothèse » tout ce qui n'est pas fait n'est cependant pas un phénomène spécifiquement anglais : les historiens des sociétés savantes françaises ont de leur côté souligné que les milieux savants des années 1660 et la première Académie des sciences chantaient aussi les louanges des faits expérimentaux tout en refusant de souscrire à quelque dogmatisme que ce soit ${ }^{95}$. Ils apparaissent, en ce sens, comme le creuset d'où provient le scepticisme expérimentaliste dont Bernard Le Bovier de Fontenelle fera une exigence institutionnelle à la fin du siècle :

«Jusqu'à présent l'Académie des sciences ne prend la nature que par petites parcelles. Nul système général, de peur de tomber dans l'inconvénient des systèmes précipités [...] Aujourd'hui on s'assure d'un fait, demain d'un autre qui n'a nul rapport. On ne laisse pas de hasarder des conjectures sur les causes, mais ce sont des conjectures. Aussi les recueils que l'Académie présente tous les ans au public ne sont composés que de morceaux détachés, et indépendants les uns des autres, dont chaque particulier, qui en est l'auteur, garantit les faits et les expériences, et dont l'Académie n'approuve les raisonnements qu'avec toutes les restrictions d'un sage pyrrhonisme ${ }^{96}$. »

Comment maintenant expliquer la coïncidence entre l'état d'esprit des savants des deux côtés de la Manche? Une première espèce d'explication a consisté à mettre en valeur l'influence de la Royal Society ou celle de Gassendi sur les sociétés savantes françaises. D'une part en effet, la Royal Society a, dès sa fondation, fonctionné pour les milieux savants français comme un modèle qu'il fallait non seulement imiter mais dépasser ${ }^{97}$.

94. À Oldenburg, 6 fév. 1671/72, in The Correspondence of Isaac Newton, éd. Herbert Westren Turnbull et al., 7 vol., Cambridge, Cambridge University Press, 1959-1977, t. I, p. $96-97$. Les notes des éditeurs, n. 18 p. 190 et n. 22 p. 386 , font le point sur l'histoire de ce passage. Z. BECHLER, op. cit. supra n. 61, montre que Hooke, Ignace-Gaston Pardies et Huygens ne s'opposent pas seulement au contenu scientifique de la lettre de Newton, mais à son style dogmatique.

95. Je me limite ici au cas français, pour ne pas alourdir un argument déjà complexe; sur le scepticisme expérimentaliste des sociétés françaises, voir Harcourt Brown, Scientific Organizations in seventeenth-century France, New York, Russel and Russel, 1934, p. 119 sqq.; John Milton Hirschrield, The Académie royale des sciences. 1666-1683, New York, Arno Press, 1981, p. 111-116; René TATON, Les Origines de l'Académie royale des sciences, conférence du palais de la Découverte, 15 mai 1965, Paris, Palais de la Découverte, 1966, p. 22, en part. n. 66 et 67; Roger HAHN, The Anatomy of a scientific institution. The Paris Academy of sciences, 1666-1803, Berkeley/Los Angeles/Londres, University of California Press, 1971, p. $30-34$.

96. Bernard Le Bovier de Fontenelle, «Préface sur l'utilité des mathématiques et de la physique et sur les travaux de l'Académie des sciences ", in CEuvres complètes, éd. Alain Niderst, Paris, Fayard, 1989-1996, t. VI, p. 47-48; également Histoire de l'Académie des sciences depuis I666 jusqu'en 1699, préf., in ibid., t. VII, p. 353.

97. Les premières histoires de l'Académie des sciences que rédigèrent Melchisédech Thévenot, Jean-Dominique Cassini et Fontenelle, arguent du passage d'Oldenburg à l'académie 
D'autre part, les instigateurs de l'Académie des sciences étaient en général plus gassendistes que cartésiens et les deux physiciens cartésiens les plus notables à l'époque, Rohault et Régis, ne furent pas choisis parmi ses premiers membres ${ }^{98}$. Mais, outre les réserves méthodologiques générales que l'on peut formuler à l'égard de la notion d'influence, ni l'influence de la Royal Society ni celle de Gassendi ne suffisent à expliquer le scepticisme expérimentaliste supposé partagé par les savants anglais et français. D'une part, les milieux savants français ont leur dynamique propre - une influence ne s'exerce pas sans l'assentiment de celui sur lequel elle s'exerce. D'autre part, et cela est souligné même par les tenants de l'influence gassendiste, l'Académie des sciences se fit de plus en plus cartésienne dans les vingt dernières années du XviI ${ }^{e}$ siècle, alors même que son scepticisme expérimentaliste allait croissant.

On comprend qu'au modèle naturaliste selon lequel les idées prolifèrent, se propagent ou s'influencent, certains historiens aient préféré un modèle plus mécanique, replaçant les idées dans leur contexte. Ce qui n'est évidemment rien dire, car, une idée n'ayant pas de contexte naturel, il faut déterminer au cas par cas pourquoi telle idée doit être placée dans ce contexte-ci plutôt que dans celui-là. L'objectif général des paragraphes suivants sera de montrer la complexité d'une analyse contextuelle du scepticisme expérimentaliste dans les milieux savants français du dernier tiers du siècle, et plus particulièrement les limites de ce qui paraît être aujourd'hui une tendance générale : insister non sur le savoir, mais sur les formes de sa communication, ce que d'aucuns appellent les règles de la civilité savante.

Montmor pour soutenir que ce sont les Français qui ont donné l'exemple aux Anglais; ce à quoi Hooke répondit qu'Oldenburg était un ouvrier de la dernière heure et que ceux qui le connaissaient «[...] understood well enough how little he himself knew of philosophick [sic] matters ", voir H. BROWN, op. cit. supra n. 95, p. 91. Toute aversion idiosynchrasique pour Oldenburg mise de côté, il faut bien reconnaître que Thévenot, Cassini et Fontenelle toilettaient l'histoire à l'avantage du parti français : les témoignages les plus directs, et en particulier la correspondance de Huygens, montrent l'attraction et l'émulation que la Royal Society exerça sur les savants français au début des années 1660; voir : Chapelain à Huygens, 30 mai 1661, in S.H.S. III, p. 273; Huygens à Moray, 24 juin 1661, in S.H.S. III, p. 284; Bouillau à Huygens, 11 juil. 1661, in S.H.S. III, p. 293; Chapelain à Huygens, 20 juil. 1661, in S.H.S. III, p. 299 ; passim.

98. Sur le rôle des gassendistes dans la formation de l'Académie voir, outre les ouvrages cités supra n. 95, L. S. JoY, op. cit. supra n. 25, p. 8-12, 209-212; Thomas M. LenNon, The Battle of the gods and giants. The legacies of Descartes and Gassendi, 1655-1715, Princeton, Princeton University Press, 1993, p. 62-106. Sur le gassendisme de Chapelain, voir Georges Collas, Jean Chapelain, 1595-1574. Étude historique et littéraire d'après des documents inédits, Paris, Perrin, 1912, en part. p. 60-64, 151-154, 331-336, 383-388. Sur le gassendisme de Sorbière, voir R. PinTARD, op. cit. supra n. 60, p. 334-348, à nuancer cependant par p. 418$420,425,429$. Que les cartésiens aient été exclus lors de la fondation de l'Académie des sciences passe aujourd'hui pour un fait historique bien établi. Il y a là en partie un mythe : le préjugé éculé selon lequel le "cartésianisme " serait par essence incompatible avec une science expérimentale et collective tient souvent lieu de preuve historique. L'espace me manque ici pour déconstruire systématiquement ce mythe; je fournis ici et là quelques éléments de cette déconstruction. 
Le scepticisme expérimentaliste a en effet été interprété comme une conséquence des contraintes liées à l'institutionnalisation de la science ${ }^{99}$. Les membres des premières sociétés savantes se seraient trouvés confrontés à un problème général : comment articuler dans une œuvre collective des contributions, sinon toujours opposées, du moins différentes au point d'engendrer de violentes querelles? L'instauration de règles d'une civilité savante aurait été une réponse à ce problème; en présentant leurs théories comme de "pures hypothèses », les physiciens en auraient atténué la force, ils auraient manifesté un certain respect pour les opinions d'autrui, et évité parfois des querelles d'amour-propre. Une telle interprétation est peut-être séduisante, mais elle appelle des précisions historiques et conceptuelles. Quels documents permettent en premier lieu de conclure au scepticisme expérimentaliste des savants français dans le dernier tiers du xvII siècle? Le scepticisme expérimentaliste doit-il en second lieu être compris comme un moyen d'éviter les querelles? Enfin, et plus généralement, si on considère le scepticisme expérimentaliste non tant comme une position épistémologique forte que comme une manière de discourir poliment, dans quel contexte intellectuel le replacer?

La première question est donc une question de fait : de quels documents disposons-nous pour établir que les milieux savants faisaient profession de scepticisme expérimentaliste dans le dernier tiers du XvII siècle? Nous nous limiterons ici à un type particulier de sources, à savoir les quelques textes où des personnages impliqués dans la fondation de l'Académie des sciences abordent directement et explicitement la question de savoir selon quels principes il faut organiser les assemblées de savants. Le premier de ces documents est un discours que Samuel Sorbière prononça en 1663 devant l'académie Montmor; son objectif était de tirer les leçons des erreurs du passé. Selon Sorbière, cette assemblée, officiellement instituée en 1657 , connut tout d'abord une période d'harmonie : « [...] il fut dit céans

99. R. HAHN, op. cit. supra n. 95, p. 30-34 et surtout L. Daston, art. cit. supra n. 92, p. 50-58. La bible sur les scientifiques anglais est S. SHAPIN, A social history of truth. Civility and science in seventeenth-century England, Chicago, Chicago University Press, 1994. Mario Biagloli, dans l'article «Le prince et les savants", Annales. Histoire, sciences sociales, $50^{\mathrm{e}}$ année, 6,1995, p. $1417-1453$, distingue différentes formes de communication en fonction de la plus ou moins grande implication du prince dans les sociétés savantes. Je cite ici seulement quelques publications; rien n'est cependant devenu plus commun que l'analyse de la « civilité savante » ou des « sociabilités scientifiques ». Il y aurait beaucoup à dire sur la signification historique, sociale et politique de cette mode universitaire. Elle trouve son origine intellectuelle dans les travaux de Norbert Elias, poursuivis en l'occurrence par Roger CHARTIER, article « CIVILITÉ », in Handbuch politish-sozialer Grundbegriffe in Frankreich 16801820, éd. Rolf ReichardT et Eberhard Schmit, Heft 4, Munich/Vienne, Oldenbourg, 1986, et par Jacques Revel, «Les usages de la civilité », in Histoire de la vie privée, t. III, éd. R. Chartier, Paris, Seuil, 1986, p. 168-209. 
d'excellentes choses, il y fut même fait plusieurs belles expériences. Et quand une matière y avait été agitée en deux ou trois séances, il semblait que l'on y avait épuisé toute la subtilité humaine ${ }^{100}$. " Mais rapidement la dissonance s'installa dans cette assemblée par la faute de «certains esprits qui s'estimèrent devoir être au-dessus des lois que nous avions établies »; dès lors, «tous les autres [...] furent comme étouffés sous les torrents de paroles ${ }^{101}$. Ce désordre fut attisé par deux espèces de personnes qui pensaient en tirer avantage : ceux qui aimaient en général les querelles parce qu'elles leur permettaient de se poser en « arbitres des savants »; ceux qui espéraient faire totalement disparaître les raisonnements au profit des expériences :

«[...] ils n'ont prêché que les expériences et ont demandé qu'on ne s'assemblât que pour en faire; ou que l'on ne discourût que sur le champ. Ils ont dit, qu'il ne fallait avoir soin que de bien agir, et qu'il n'était pas besoin de raisonner sur aucune matière avant qu'on eût fait quelque expérience, ce qui fournirait assez de sujet de bien parler sans autre méditation ${ }^{102}$. "

100. «Discours prononcé le 3 d'avril 1663. À l'ouverture de l'Académie de physiciens qui s'assemblent tous les mardis chez monsieur de Montmor», in Guillaume BIGOURDAN, «Les premières réunions savantes de Paris au xvil siècle ", Comptes rendus hebdomadaires des séances de l'Académie des sciences, t. 164, séance du 22 janv. 1917, p. 160-161. Le fil directeur du discours de 1663 est qu'une assemblée physique a besoin à la fois de discours et d'expériences. Il n'était pourtant pas question d'expériences dans le « Règlement de l'assemblée de physiciens, qui se fit à Paris, chez monsieur de Montmor l'an 1657 ", rédigé par Sorbière et Abraham Du Prat : il avait pour objet seulement les discours, et plus précisément des discours écrits par avance (nous connaissons le règlement de 1657 par la lettre de Sorbière à Hobbes, $1^{\text {er }}$ fév. 1658, publiée in G. Bigourdan, op. cit., séance du 15 janv. 1917, p. 129-133, également accessible in S.H.S. IV, p. 513). Le premier règlement d'une société savante française à faire place aux expériences, aux instruments et aux machines, est à ma connaissance le "Projet de la compagnie des sciences et des arts » rédigé en 1663 par Huygens, in S.H.S. IV, p. 325 .

101. "Discours prononcé le 3 d'avril 1663 [...] ", cité supra n. 100, p. 161. Parmi ceux qui furent étouffés, Sorbière signale plus particulièrement deux esprits " pénétrants et judicieux », « le savant M. Du Prat et l'agréable M. Du Bosc ". Il ne nomme pas les deux personnes qui les étouffaient; le témoignage de Huygens incite à penser qu'il s'agissait de Roberval et d'Antoine de La Poterie. Huygens voit en effet dans l'impair commis par le premier (qu'Ismaël Boulliau lui avait raconté dans la lettre du 6 déc. 1658, in S.H.S. II, p. 267) l'origine du languissement de l'académie Montmor (voir S.H.S. II, p. 468). Il avait d'autre part noté dans son journal de voyage que La Poterie contredisait Girard Desargues « avec une véhémence merveilleuse et ridicule " (9 nov. 1660, in S.H.S. XXII, p. 535); deux ans après son passage à Paris, il répondra à son frère Lodewijk, 26 avr. 1662, in S.H.S. IV, p. 117, qui lui mentionnait les outrances de La Poterie : « J'y [à l'académie Montmor] ai souvent entendu cet aristotélicien qui dispute avec tant de furie, et, s'il eût dépendu de moi, je l'aurais banni de là. "

102. "Discours prononcé le 3 d'avril 1663 [...] 》, cité supra n. 100, p. 162. Là encore, on souhaiterait mettre des noms derrière ces catégories générales. Sorbière indique que Rohault, Jean Pecquet, Pierre Petit, Thévenot et Balthazar de Montconys vinrent présenter diverses expériences à l'académie Montmor; voir séance du 29 janv. 1917, in ibid., p. 216-217. On peut cependant « faire des expériences " sans pour autant " ne prêcher que des expériences ». 
Conséquemment, la recommandation essentielle de Sorbière pour une académie scientifique est-elle de mélanger expériences et raisonnements, tout en favorisant un certain éclectisme théorique :

« Il est fort naturel à chacun de faire valoir son talent $[\ldots]$ : ceux qui [...] ont vaqué à quelques expériences, ne parlent que des expériences; ceux qui ont de la facilité à bien parler [...] ne demandent que des entretiens; et ceux qui ont quelque faculté à digérer par écrit des matières difficiles sont bien aises que cette méthode ne soit pas tout à fait négligée. Chacun veut faire gagner céans sa secte, ses principes, ou son hypothèse; et il y en a peu qui prennent plaisir à y entendre les divers styles des philosophes, ou les diverses pensées que l'on y produit sur un même sujet : quoique cette variété peut servir d'ornement, et que les sceptiques, qui aiment fort à écouter, ne trouvassent rien plus agréable que cette symphonie composée d'un péripatéticien, d'un lulliste, d'un cartésien, d'un chimiste, d'un platonicien, d'un ami de Lucrèce, et de quelques autres philosophes, qui sur un même ton, c'est-à-dire avec le même dessein de trouver la vérité, chanteraient des paroles différentes, et feraient des passages et des roulements fort différents. Il serait à désirer, Messieurs, qu'on n'exclût aucune méthode, pourvu qu'elle ne contrevint pas à l'ordre; et que chacun écoutât dans le plus profond silence celle de son compagnon, afin qu'il fut écouté de même à son tour. Et ainsi j'estime qu'il faudrait faire place aux expériences, et se taire lorsque quelqu'un voudrait parler ${ }^{103}$.»

Le discours de Sorbière est incontestablement empreint de ce qu'on peut appeler «scepticisme expérimentaliste "; le problème est cependant de déterminer jusqu'où il peut être considéré comme représentatif de l'état d'esprit des milieux savants. L'autre document majeur que nous possédons sur l'établissement de l'Académie des sciences est un projet que Jean Chapelain rédigea en 1666 à l'intention de Colbert. Chapelain avait comme Sorbière fréquenté l'académie Montmor, et il avait plus d'une fois eu l'occasion de se plaindre des «digladations entre les philosophes et les mathématiciens ${ }^{104} \%$. Il entend lui aussi prendre modèle sur les assemblées ayant existé, à ceci près évidemment qu'une Académie instaurée par Louis XIV sera « incomparablement plus be[lle] que ce qu'on a jamais vu en ce genre ${ }^{105} »$. Mais le seul problème pour Chapelain consiste à bien choisir les membres de la future Académie. Son conseil est simple : il faut

103. Ibid., séance du 29 janv. 1917, p. 216.

104. Chapelain à Heinsius, 6 fév. 1659, in Lettres de Jean Chapelain, op. cit. supra n. 63 , t. II, p. 17.

105. «Lettre de M. Chapelain à M. Colbert dans lesquelles il expose son sentiment sur les divers projets de création et d'encouragement que méditait le ministre en faveur des arts et des sciences ", in Lettres, instructions et mémoires de Colbert, éd. Pierre Clément, 8 vol., Paris, Perrin, 1861-1882, vol. V, p. 513. G. Collas, in op. cit. supra n. 98, p. 384-388, établit que Chapelain est l'auteur de cette lettre. 
se garder d'écouter ceux qui s'occupent plus à fomenter des cabales qu'à cultiver la vérité :

«Il y a dans le monde des savants de deux sortes. Les uns s'adonnent aux sciences, parce qu'elles leur plaisent; ils se contentent pour tout fruit de leurs travaux des connaissances qu'ils acquièrent, sans affecter de les publier [...] Ceux-là sont des savants de bonne foi [...]. Il y en a d'autres qui ne cultivent les sciences que comme un champ qui les doit nourrir; et comme ils voient par expérience que les grandes rétributions ne se donnent qu'à ceux qui font le plus de bruit dans le monde, ils s'appliquent particulièrement, non pas à faire de nouvelles découvertes, [...] mais à tout ce qui les peut faire paraître; ils affectent surtout d'être connus à la cour; ils font des cabales pour cela [...] Ceux-là sont les savants du beau monde ${ }^{106}$.»

Si on prête l'oreille à ces «savants de profession », continue Chapelain, « il arrivera indubitablement que, par intérêt et par prévention, ils excluront tous ceux dont ils appréhendent la capacité ou qui ne sont pas de leur opinion ». Si, par contre, on s'en rapporte "à des personnes d'une qualité assez éminente pour être au-dessus de toutes ces faiblesses ", elles sauront distinguer « les différents génies de ceux qui sont propres à faire une expérience ou de ceux qui en savent tirer toutes les utilités, de ceux qui ont assez de netteté d'esprit pour les recueillir et enfin de tous ceux qui ont les différents talents dont l'assortiment pourrait rendre une académie royale aussi belle qu'utile ». Plus que chez Sorbière, les expériences, et plus exactement les expériences utiles, sont au cœur du projet de Chapelain; de fait, l'objectif de la future Académie ne sera rien moins que

«[...] de bannir des sciences tous les préjugés, en ne s'appuyant que sur des expériences, d'y trouver quelque chose de certain, d'en écarter toutes les chimères et d'ouvrir à ceux qui les cultivent à l'avenir un chemin aisé à la vérité, autant que Dieu a donné aux hommes de la connaître pour leur utilité ${ }^{107} \gg$.

Une fois la part faite des lieux communs dans ces deux projets - c'est par exemple en homme de salon et de cour, rompu aux flatteries et expert en cabales, que Chapelain pourfend les savants du beau monde -, et une fois rappelé que leurs auteurs, s'ils s'intéressaient incontestablement aux sciences, n'étaient tout de même pas de grands savants, il faut reconnaître qu'ils font confiance aux expériences et refusent de prendre parti pour un

106. «Lettre de M. Chapelain à M. Colbert [...] », in op. cit. supra n. 105, p. 514.

107. Ihid. 
système. En ce sens, on peut parler de « scepticisme expérimentaliste »; que, maintenant, le scepticisme expérimentaliste ait été une réponse au problème des querelles savantes, c'est ce qui paraît difficile à établir. Il est vrai que les querelles étaient nombreuses entre savants, et que tout manquement à la civilité était condamné. L'exemple le plus fameux en France à cette époque est sans doute la « sottise » que commit Roberval chez monsieur de Montmor :

" [...] il a été si incivil que de lui dire dans sa maison, s'étant piqué sur une des opinions de monsieur Descartes que monsieur de Montmor approuvait, qu'il avait plus d'esprit que lui et qu'il n'avait rien de moins que lui que le bien et la charge de maître des requêtes, et que s'il était maître des requêtes, qu'il [sic] vaudrait cent fois mieux que lui. Monsieur de Montmor qui est très sage lui dit, qu'il en pourrait et devrait user plus civilement, que de le quereller et le traiter de mépris dans sa maison. Toute la compagnie trouva fort étrange la rusticité et la pédanterie de monsieur de Roberval ${ }^{108}$."

Dans son discours de 1663, Sorbière stigmatise la confusion des assemblées de physique : «Elle fait honte à de si nobles études; car ce n'est presque qu'en celles de physique que je la vois régner, et partout ailleurs on s'écoute et on laisse parler ceux qui ont commencé à dire quelque chose ${ }^{109}$. " Plus directement, Ismaël Boulliau oppose l'urbanité des littéraires réunis autour de l'ambassadeur de Venise à la véhémence des montmoréens :

« À ce qu'on m'en a dit, les Vénitiens sont plus agréables, plus policés et plus urbains, et dans leurs discussions ils usent de compliments. Les montmoréens sont plus tranchants et ils s'entretiennent avec violence, comme ceux qui sont en désaccord à propos de la vérité qu'ils poursuivent : parfois c'est même avec plaisir qu'ils se raillent et avec jalousie qu'ils se reprennent; c'est la vérité qu'ils prétendent tous chercher et examiner, mais chacun voudrait en être le seul auteur une fois qu'elle est découverte ${ }^{110}$."

Il est cependant difficile de croire que le principal avantage du scepticisme expérimentaliste ait été d'éviter les querelles. Fait-on d'ailleurs vraiment preuve de civilité à tout plonger dans la nuit indistincte des hypothèses probables? Lorsque Paul Boccone, herboriste de feu le grand duc de Toscane, vint à Paris en 1669 pour instituer une conférence sur la nomen-

108. Boulliau à Huygens, 6 déc. 1658, in S.H.S. II, p. 267.

109. «Discours prononcé le 3 d'avril 1663 [...]», cité supra n. 100 , p. 161.

110. Boulliau à Heinsius, fév. 1658, in H. BROwN, op. cit. supra n. 95, p. 78-79. 
clature des plantes, il précisa qu'on pouvait, sans manquer à la civilité, soit présenter ses opinions comme probables, soit les présenter comme certaines :

«On [...] parlera avec modestie de la manière suivante : cette plante me paraît être nommée N. par tel auteur, toutefois je n'en suis pas assuré, je vous prie Messieurs, d'examiner ma conjecture; ou bien, j'ai examiné cette plante, et j'estime absolument que c'est $\mathrm{N}$. décrite par un tel auteur; cependant chacun peut écrire ou assurément ou avec doute son opinion, sans craindre d'être repris. On trouvera dans ma chambre des livres de botanique, pour confronter la plante avec la figure ou description d'icelles, et puis on établira ce qu'il y a de certain, le tout avec honneur et respect les uns envers les autres, et le plus de modestie que faire se pourra ${ }^{11 !}$.»

Ce qui, cependant, peut retenir de rejeter totalement la prise en compte des règles de communication du savoir, c'est l'importance au $\mathrm{XVII}^{\mathrm{e}}$ siècle de certains aspects de la culture humaniste qui ont parfois été regroupés sous le nom de «probabilisme cicéronien ${ }^{112}$ ». Aussi esquisserons-nous pour finir un vaste programme, celui de l'histoire de la prise en compte de certaines valeurs humanistes par les savants ${ }^{113}$. La revalorisation de la dialectique opérée par des auteurs comme Lorenzo Valla, Rudolph Agricola ou Pierre de La Ramée (dit Ramus) avait eu valeur de machine de guerre contre la conception qu'avaient les scolastiques de l'objet du savoir, de la manière de l'obtenir et de le communiquer : ces humanistes ne considéraient pas la dialectique comme la voie royale vers une vérité absolue, mais comme un moyen de recenser des opinions probables dont les hommes doivent le plus souvent se contenter; à la démonstration syllogistique, ils avaient préféré l'argumentation in utramque partem, qui n'admet pas toujours de conclusion, ou l'épicheirema qui est une espèce de syllogisme

111. «Avis aux personnes d'esprit, et aux curieux [...] », in H. Brown, op. cit. supra n. 95, p. 284.

112. L'expression "probabilisme cicéronien " vient de l'article de C. J. R. ArmSTrong, "The dialectical road to truth : the dialogue ", in French Renaissance Studies. 1540-1570, éd. Peter Sharatr, Edimbourg, Edinburgh University Press, 1976, p. 36-51, qui met en lumière le lien entre le genre littéraire du dialogue et une conception sceptique de la dialectique. Peter DEAR a introduit cette catégorie en histoire des sciences dans " Marin Mersenne and the probabilistic roots of "mitigated scepticism" ", Journal of the history of philosophy, vol. 22, 2, 1984, repr. in Mersenne and the learning of the Schools, Ithaca, NJ, Cornell University Press, 1988, p. 26-47.

113. Peu de travaux ont à ma connaissance étudié la confrontation des valeurs humanistes et savantes au XVII siècle. Voir cependant Roger ZUBER, « L'humanisme et les savants : de Peiresc aux Perrault », Revue française d'histoire du livre, 1983, p. 33-51 ; B. C. Southgate, " "No other Wisdom"? Humanist reactions to science and scientism in the seventeenth century », The Seventeenth Century, 5, 1990, p. 71-92. On trouve aussi des éléments de réflexion in R. PINTARD, op. cit. supra n. 60, et in L. S. JoY, op. cit. supra n. 25, en part. p. 8-12, 209212. 
incomplet ${ }^{114}$. Certains dialogues littéraires de la Renaissance sont imprégnés de ce probabilisme cicéronien; il constituait également un arrière-plan théorique des conversations entre honnêtes gens ${ }^{115}$.

À partir du xvI ${ }^{e}$ siècle, la conversation est souvent présentée comme le plus vif ou le plus doux des plaisirs que les hommes puissent trouver en société ${ }^{116}$; lorsqu'elle a pour objet le vrai, elle est opposée à l'étude des livres, parce qu'elle ne rebute pas les esprits, mais les stimule et les échauffe ${ }^{117}$. Elle est également distinguée de la disputatio scolastique, qui repose sur une conception dogmatique du savoir et dégénère le plus souvent en conflit. Ainsi Théophraste Renaudot critique-t-il les mauvaises manières des scolastiques, leurs « disputes et contradictions n'offusquant pas seulement toute la grâce et le plaisir de l'entretien, mais finissant même en riotes et injures pédantiques ${ }^{118} »$; en apprenant à ne jamais céder, les jeunes gens développent selon lui une «humeur opiniâtre », "l'une des plus désobligeantes qualités et la plus inepte en compagnie ${ }^{119}$. Les rituels

114. Sur la dimension philosophique de la rhétorique cicéronienne, voir Alain MiCHEL, Les Rapports de la rhétorique et de la philosophie dans l'auvre de Cicéron. Recherches sur les fondements philosophiques de l'art de persuader, Paris, Presses universitaires de France, 1960, en part. chap. III, p. 158-234. Sur l'utilisation humaniste de Cicéron contre la scolastique, voir Lisa JARDINE, "Lorenzo Valla and the intellectual origins of humanist dialectic ", Journal of the history of philosophy, vol. 15, 1977, p. 143-164.

115. Sur la signification de la forme du dialogue dans l'Italie de la Renaissance, voir David MARSH, The Quattrocento dialogue. Classical tradition and humanism innovation, Londres/ Cambridge, MA, Harvard University Press, 1980, et Virginia Cox, The Renaissance dialogue. Literary dialogue in its social and political context, Cambridge, Cambridge University Press, 1992. Sur l'histoire de la conversation et de son mythe, voir Marc FuMAROLI, « La conversation ", in Les Lieux de mémoire française, éd. Pierre Nora, vol. III, t. 3, Paris, Gallimard, 1993, p. 679-743; M. FumARoLI, «La conversation savante», in Commercium Litterarium. 1600-1750, éd. Hans Bots et Françoise WAQUET, Amsterdam/Maarsen, APA Holland University Press, 1994.

116. Stefano Guazzo, La Civil Conversazione, Venise, 1579, p. 168, cité in V. Cox, op. cit. supra n. 115, p. 22; Chevalier DE MÉRÉ, Les Conversations, «Quatrième conversation », in CEuvres complètes, éd. Charles H. Boudhors, Paris, Éd. Fernand Roches, 1930, t. I, p. 51.

117. Les références suivantes ne peuvent être qu'arbitraires, puisqu'elles portent sur deux siècles. Montaigne, Essais, liv. III, chap. vili, op. cit. supra n. 70, p. 894; SPRAT, HRS, p. 98 ; LeIbniz, à L'Hôpital, 23 mars 1699, in Die mathematische Schriften, éd. C. I. Gerhard, Berlin/Halle, 1849-1863, repr. Hildesheim, Georg Olms, 1971, vol. II, p. 334; Daniel-Georges MoRHof, Polyhistor sive de notitia auctorum et rerum commentarii [...], Lübeck, 1688, chap. $\mathrm{xv}$, "De conversatione erudita», p. 153, cité in M. Fumarol, «La conversation savante ", art. cit. supra n. 115 , p. 75 ; passim.

118. Première centurie des questions traitées es conférences du Bureau d'Adresse [...], Avis au lecteur, Paris, 1634, cité in Simone MAZAURIC, « La diffusion du savoir en dehors des circuits savants. Le bureau d'adresse de Théophraste Renaudot ", in Commercium Litterarium. 1600-1750, op. cit. supra n. 115, p. 156.

119. Quatrième centurie des questions traitées es conférences du Bureau d'Adresse [...], Paris, 1641, p. 420; cité in ibid., p. 156. Sur la condamnation des disputes scolastiques, voir, là encore arbitrairement, DESCARTES, $D M$, sixième partie, in A.T. VI, p. 70-71; GASSENDI, $D P$, in Rochot, 1959 , p. 26-27, 48-49, 52-55; Glanvill, VD, chap. XXVII, in G.C.W. I, p. 159-168; Oldenburg, à Pierre de Carcavy, in The Correspondence of Henry Oldenburg, éd. A. Rupert Hall et Marie Boas-Hall, 13 vol., Madison/Londres, Wisconsin University Press, 1965-1986, t. IV, p. 101 ; SPRAT, HRS, p. 17-18, 429; passim. 
de déférence dans une conversation entre honnêtes gens sont multiples : on soulignera que prendre la parole est une fatalité plutôt qu'un choix, on dépréciera ses qualités d'orateur, on évitera de monopoliser la parole : ce serait considérer son interlocuteur comme un élève à instruire, non comme un compagnon sur la route du vrai ${ }^{120}$. Quoique la contradiction soit le ressort des conversations fécondes, on la manipulera avec tact :

"Pour contredire une personne de qualité, il ne faut jamais faire crûment, par un "Non, Monsieur, cela n'est pas ainsi", mais par une circonlocution en défaut, par exemple: "Vous me pardonnerez, Monseigneur, Je vous demande pardon, Madame, si j'ose dire que" ${ }^{12 !}$. »

Il y a peut-être en ce sens à se demander jusqu'où les valeurs humanistes ont marqué la manière d'écrire des savants; mais un tel travail ne saurait cependant se dispenser d'un souci épistémologique ou faire l'économie d'études locales. En effet, l'idéal humaniste n'est ni une chose naturelle, toujours déjà donnée, ni une chose matérielle, qui serait transportée ici ou là sans modification, mais une valeur sociale et culturelle, autrement dit quelque chose que les hommes transforment, ou du moins réinvestissent d'un sens nouveau dans différents contextes. Or rien ne nous garantit tout d'abord que la science soit une matière à discussion comme la question de savoir ce que sont les qualités du bon courtisan ou du parfait amant. Pour Aristote, Cicéron et les humanistes eux-mêmes, le savoir des choses naturelles était une science et la rhétorique commençait là où la science finissait; en ce sens, l'application de la catégorie rhétorique du probable à la physique constitue une rupture épistémologique qu'on ne peut passer sous silence. De plus, les différents domaines de la science des choses naturelles n'étaient pas soumis au même régime. Proposer des explications mécaniques à titre d'hypothèses était normal, mais les propositions de la science du mouvement étaient des théorèmes. Dans le passage de la lettre sur les couleurs qu'Oldenburg censura, Newton prend le contre-pied de ce qu'il juge être la position épistémologique la plus commune des philosophes naturels non pas en général, mais à propos d'une discipline particulière : la partie de l'optique qui traite des couleurs. De fait, les membres de la Royal Society furent choqués qu'il présentât son hypothèse sur les couleurs sur le mode dogmatique, mais ils ne le furent pas lorsqu'il présentât ainsi un traité de mécanique.

120. Voir, sur ce demier point, les textes de Giovanni Della Casa, Stefano Guazzo, Sperone SPERONI et Torquato TAsso, cités in V. Cox, op. cit. supra n. 115, p. 41, 43, 153. Sur les rituels de déférence en général, voir les remarques de V. Cox, in ibid., p. 40-46.

121. Antoine DE CourTin, Nouveau Traité de civilité, Bruxelles, 1650 , p. 50, cité in M. BiAGIOLI, op. cit. supra n. 99 , p. 1421, n. 16. Sur la fécondité de la contradiction, voir le texte de Montaigne indiqué n. 117 et les remarques de V. Cox, op. cit. supra n. 115, p. 157-158. 
Les deux premières parties de cet article avaient admis que, lorsqu'un physicien appelait «hypothèse " une proposition, il se prononçait sur le degré de certitude qu'il pouvait lui assigner par rapport à d'autres énoncés scientifiques. Dans la troisième partie, une idée bien différente a été éprouvée: l'introduction d'hypothèses en physique résulterait de contraintes imposées, non par le savoir, mais par les règles de sa communication. Aussi s'est-elle concentrée sur les quelques années qui précédaient la fondation de l'Académie des sciences, précisément parce qu'il s'agissait d'un moment d'interrogations, de récapitulation du passé et de réflexion sur l'avenir. Le résultat de cette enquête est à la fois positif en général et négatif en particulier : il est certain que la question de la communication du savoir préoccupait les milieux savants; il est impossible de prouver qu'ils jugeaient que l'emploi d'un langage hypothétique pouvait en favoriser l'effectuation. En un mot, il semble que, quelle que soit l'importance qu'on veuille accorder aux règles de communication du savoir, elles ne suffisent pas à rendre compte de la différence entre le certain et le probable - de même les règles de la civilité représentent, mais ne produisent pas, la différence entre le noble et l'ignoble.

Cet article s'était initialement proposé deux objectifs indissociables : montrer que la catégorie de scepticisme modéré définie par Popkin dans le contexte d'une histoire du scepticisme ne pouvait être intégrée telle quelle en histoire des sciences; déterminer comment des historiens pouvaient rendre compte de l'introduction d'hypothèses dans la physique du XVII' siècle. Nous avons à cet effet adopté successivement trois perspectives : celle d'un historien de la philosophie, suivant pas à pas la signification du concept d'hypothèse chez Descartes; celle d'un historien de la méthodologie scientifique, montrant la corrélation historique entre l'emploi que les philosophes mécaniques font de la notion d'hypothèse et leurs convictions ontologiques; celle d'un historien faisant du recours aux hypothèses une question de civilité.

Trois brèves remarques sur ces trois perspectives s'imposent maintenant. En premier lieu, elles n'auraient pu être adoptées à propos de n'importe quel sujet: la question des hypothèses a cette particularité qu'elle ne touche pas principalement à la production d'énoncés physiques, mais à la manière dont ils sont désignés ou réfléchis dans un métalangage qui possède une certaine autonomie par rapport aux exigences de la physique comme science. En deuxième lieu, ces trois perspectives ne doivent pas être confondues; il serait par exemple bon de réserver le terme "épistémologie » à des systèmes consistants d'énoncés théoriques sur la science comme cela a été rappelé au passage dans la première partie, l'usage du 
mot « hypothèse » ne suffit pas à mettre en œuvre une "épistémologie ". Enfin, elles n'autorisent pas le même degré d'exactitude : l'entité « Descartes " est plus facile à contrôler que l'entité "philosophie mécanique " - pour ne rien dire des "savants français de la fin du xvir siècle ».

Au terme de ce parcours, il faut revenir sur la spécificité du scepticisme. Les historiens du scepticisme tiennent à réserver le terme "sceptique » aux textes recourant à une argumentation inspirée des Académiques de Cicéron ou des Hypotyposes de Sextus pour défendre la thèse générale qu'il est impossible de parvenir à un savoir certain ${ }^{122}$. Il n'empêche que le scepticisme présente une particularité par rapport à d'autres traditions philosophiques: l'argumentation sceptique peut être employée hors de son contexte d'origine, et il existe des attitudes sceptiques indépendantes de la tradition philosophique sceptique. C'est sans doute cette spécificité qui a permis à Popkin de se placer en un point où pouvaient se rencontrer non seulement l'historien de la philosophie, des méthodologies scientifiques ou des règles de communication du savoir, mais tout aussi bien celui de la pensée politique ou religieuse. Il nous reste, par-delà des histoires disciplinaires, à tenter de retrouver, ou peut-être seulement de comprendre, l'espèce de généralité qui a animé la pensée du xvir siècle.

Sophie Roux (mars 1998).

122. $H S$, p. $57,60,63$, passim. Également C. B. SсHмrT, op. cit. supra n. 62, p. 5-6. 